\title{
Ergodic coactions with large multiplicity and monoidal equivalence of quantum groups
}

\author{
By Julien Bichon ${ }^{(a)}$, An De RiJdT ${ }^{(b)}$ And StefaAn VAes ${ }^{(b, c)}$ \\ Communications in Mathematical Physics 262 (2006), 703-728.
}

(a) Laboratoire de Mathématiques Appliquées; Université de Pau et des Pays de l'Adour; IPRA; Avenue de l'Université, F-64000 Pau (France)

(b) Department of Mathematics; K.U.Leuven; Celestijnenlaan 200B; B-3001 Leuven (Belgium)

(c) Institut de Mathématiques de Jussieu; Algèbres d'Opérateurs; 175, rue du Chevaleret; F-75013 Paris (France)

e-mail: julien.bichon@univ-pau.fr, an.derijdt@wis.kuleuven.ac.be,vaes@math.jussieu.fr

\begin{abstract}
We construct new examples of ergodic coactions of compact quantum groups, in which the multiplicity of an irreducible corepresentation can be strictly larger than the dimension of the latter. These examples are obtained using a bijective correspondence between certain ergodic coactions on $\mathrm{C}^{*}$-algebras and unitary fiber functors on the representation category of a compact quantum group. We classify these unitary fiber functors on the universal orthogonal and unitary quantum groups. The associated $\mathrm{C}^{*}$-algebras and von Neumann algebras can be defined by generators and relations, but are not yet well understood.
\end{abstract}

\section{Introduction}

By a well known theorem of Høegh-Krohn, Landstad and Størmer [16], compact groups only admit ergodic actions on tracial $\mathrm{C}^{*}$-algebras. Indeed, the (unique) invariant state is necessarily a trace. Moreover, given a compact group $G$ acting ergodically on a $C^{*}$-algebra $B$, one studies the so-called spectral subspaces: the action of $G$ on $B$ yields a unitary representation of $G$ which can be decomposed into a direct sum of irreducible representations. One proves that the multiplicity of an irreducible representation is necessarily bounded by the dimension of this irreducible representation.

A deeper analysis of the spectral structure of ergodic actions of compact groups has been made by A. Wassermann $[27,28,29]$. In the culmination of his work, Wassermann shows that the compact group $\mathrm{SU}(2)$ only admits ergodic actions on von Neumann algebras of finite type I.

In the 1980's, Woronowicz introduced the notion of a compact quantum group and generalized the classical Peter-Weyl representation theory. Many fascinating examples of compact quantum groups are available by now: Drinfel'd and Jimbo [14, 17] introduced the q-deformations of compact semi-simple Lie groups and Rosso [21] showed that they fit into the theory of Woronowicz. The universal orthogonal and unitary quantum groups were introduced by Van Daele and Wang [25] and studied in detail by Banica [2, 3]. Other examples of compact quantum groups, related with graphs and metric spaces have been constructed by the first author [9] and by Banica $[4,5]$.

The abstract theory of ergodic coactions of compact quantum groups on $\mathrm{C}^{*}$-algebras has been initiated by Boca [10] and Landstad [18]. The major difference with the compact group case, is the following: the (unique) invariant state is no longer a trace. Indeed, Wang [26] gave examples of ergodic coactions of universal unitary quantum groups on type III factors.

Moreover, in the work of Boca, the multiplicity of an irreducible corepresentation is proved to be bounded by the quantum dimension rather than the ordinary dimension of the corepresentation. Nevertheless, in all the examples known up to now, the multiplicity is actually bounded by the ordinary dimension. In this paper, we provide examples of ergodic coactions where the multiplicity of an irreducible corepresentation is strictly larger than the ordinary dimension of the corepresentation.

In [29], A. Wassermann gives a complete classification of the ergodic actions of SU(2), essentially labeling them by the finite subgroups of SU(2). It would, of course, be great to give a complete classification of ergodic coactions of the deformed $\mathrm{SU}_{q}(2)$. In [23], Tomatsu provides a first step in this direction: he computes all 
ergodic coactions of $\mathrm{SU}_{q}(2)$ on 'virtual' quotient spaces $\mathrm{SU}_{q}(2) / \Gamma$. (More precisely, he describes all the coideals of the quantum group $\mathrm{SU}_{q}(2)$.) By construction, the ergodic coactions of $\mathrm{SU}_{q}(2)$ on its virtual quotient spaces are such that the multiplicity of an irreducible corepresentation is bounded by its dimension. The results of this paper imply in particular that there are much more ergodic coactions of $\mathrm{SU}_{q}(2)$ than the ones studied by Tomatsu.

The major tool to produce our new examples of ergodic coactions of compact quantum groups, is the notion of monoidal equivalence of quantum groups. One can look at a compact quantum group with several degrees of precision. At first, we study only the fusion rules in the representation theory: we label the irreducible corepresentations and describe how a tensor product of irreducibles breaks up into irreducibles. Taking into account only these fusion rules, we loose a lot of information: for example, the $q$-deformed compact Lie groups have the same fusion rules as their classical counterparts. In a next approximation, one studies the corepresentation theory of a compact quantum group as a monoidal category, but without its concrete realization (the so-called forgetful functor to the category of Hilbert spaces). This is crucial: by the Tannaka-Krein reconstruction theorem [32], the concrete monoidal category of (finite-dimensional) corepresentations essentially determines the compact quantum group. Note that knowing the representation theory of a compact quantum group as a monoidal category, comes down to knowing the fusion rules together with the $6 j$-symbols, see Remark 3.3 .

Closely related to the notion of monoidally equivalent quantum groups, is the notion of a unitary fiber functor on a compact quantum group. Essentially, a unitary fiber functor gives another concrete realization, different from the tautological realization, of the representation theory of a compact quantum group.

In this paper, we choose not to use the abstract language of categories. We give 'down-to-earth definitions' of monoidally equivalent quantum groups and unitary fiber functors, see 3.1 and 3.7. This makes the construction of associated $\mathrm{C}^{*}$-algebras and coactions straightforward. This concrete approach is well adapted to the language of corepresentations of compact quantum groups. In this way, using previous results of Banica $[2,3]$, we can show very easily as well the monoidal equivalence of the universal orthogonal and universal unitary quantum groups.

The results in this paper can be summarized as follows.

- In Section 2, we recall the theory of spectral subspaces $[10,18]$ and provide a simple proof for the multiplicity bound. We also introduce the notion of quantum multiplicity of an irreducible corepresentation in an ergodic coaction and this can be strictly larger than the ordinary multiplicity.

- In Theorem 3.9, we show that there is a natural bijective correspondence between certain ergodic coactions of compact quantum groups and unitary fiber functors. These coactions are called of full quantum multiplicity. These are precisely the ergodic coactions for which the crossed product is isomorphic with the compact operators, see [18]. They can also be described as the ergodic coactions for which the inequality between quantum multiplicity and quantum dimension, becomes an equality.

- In Section 4, we study the special case of unitary fiber functors preserving the dimension. This leads to a bijective correspondence with unitary 2-cocycles on the dual, discrete, quantum group. The ideas for this section come from the work of Wassermann [28].

- In Sections 5, we establish the monoidal equivalence between the universal orthogonal quantum groups $A_{o}(F)$. Recall that, for any $F \in \mathrm{GL}(n, \mathbb{C})$ satisfying $F \bar{F}= \pm 1$, one defines the compact quantum group $A_{o}(F)$ as the universal quantum group generated by the coefficients of a unitary $n$ by $n$ matrix $U$ with relations $U=F \bar{U} F^{-1}$. The comultiplication on $A_{o}(F)$ is (uniquely) defined in such a way that $U$ becomes a corepresentation. We show that $A_{o}\left(F_{1}\right)$ is monoidally equivalent with $A_{o}\left(F_{2}\right)$ if and only if the signs of the $F_{i} \overline{F_{i}}$ agree and $\operatorname{Tr}\left(F_{1}^{*} F_{1}\right)=\operatorname{Tr}\left(F_{2}^{*} F_{2}\right)$. In particular, if $0<q \leq 2-\sqrt{3}$, there is a continuous family of non-isomorphic $A_{o}(F)$ monoidally equivalent with $\mathrm{SU}_{q}(2)$.

- In Section 6, we prove similar results for the universal unitary quantum groups $A_{u}(F)$, defined as the universal quantum group generated by the coefficients of a unitary $n$ by $n$ matrix $U$ with the relation that $F \bar{U} F^{-1}$ is unitary. Again, the comultiplication is defined such that $U$ becomes a corepresentation. We show that the quantum dimension of $U$, i.e. $\sqrt{\operatorname{Tr}\left(F^{*} F\right) \operatorname{Tr}\left(\left(F^{*} F\right)^{-1}\right)}$, is a complete invariant for the $A_{u}(F)$ up to monoidal equivalence. 
- Using the previous results, we obtain a complete classification of the ergodic coactions of full quantum multiplicity of $A_{o}(F)$ and $A_{u}(F)$, as well as a computation of the 2-cohomology of their duals (Corollary $5.9)$. In particular, we construct ergodic coactions of $\mathrm{SU}_{q}(2)$ such that the multiplicity of the fundamental corepresentations is arbitrarily large (Corollary 5.8).

In the theory of Hopf algebras, ergodic coactions of full quantum multiplicity correspond to Hopf-Galois extensions. In this algebraic setting, several results related to ours have been obtained. The relation between Hopf-Galois extensions and fiber functors is due to Ulbrich [24] and the relation between monoidal equivalence of Hopf algebras and Hopf-bi-Galois extensions has been established by Schauenburg [22]. Fiber functors preserving the dimension and 2-cocycles have been studied by Etingof and Gelaki [15]. The main difference between these Hopf algebraic results and our work, lies in dealing with the ${ }^{*}$-structure and positivity. In a sense, we are dealing with the real forms of (certain) Hopf algebras. This allows us to construct Hilbert space representations and $\mathrm{C}^{*}$-algebras. The compatibility of fiber functors with ${ }^{*}$-structures is a severe restriction. Indeed, there exist many fiber functors on the representation category of $\operatorname{SU}(2)$ (see [11]), but the forgetful functor is the only one compatible with the ${ }^{*}$-structure.

After completion of a first version of this paper - signed by the last two authors - the first author joined the project and his preprint [6] was taken into account, yielding the current paper as a final result.

\section{Preliminaries}

Notation 1.1. Consider a subset $S$ of a $\mathrm{C}^{*}$-algebra. We denote by $\langle S\rangle$ the linear span of $S$ and by $[S]$ the closed linear span of $S$.

The symbol $\otimes$ denotes tensor products of Hilbert spaces, minimal tensor products of $\mathrm{C}^{*}$-algebras as well as algebraic tensor products of *-algebras.

If $A$ is a *-algebra and $U \in M_{n_{1}, n_{2}}(\mathbb{C}) \otimes A$, we denote by $\bar{U}$ the matrix $\bar{U}_{i j}=U_{i j}^{*}$.

We make use of the leg numbering notation. For instance, if $v \in A \otimes B$, then $v_{12}$ denotes the element in $A \otimes B \otimes C$ defined by $v_{12}=v \otimes 1$. We analogously use the notations $v_{13}, v_{23}$, etc.

Definition 1.2. A compact quantum group $\mathbb{G}=(A, \Delta)$ consists of a unital $\mathrm{C}^{*}$-algebra $A$ together with a unital *-homomorphism $\Delta: A \rightarrow A \otimes A$ satisfying the coassociativity relation

$$
(\iota \otimes \Delta) \Delta=(\Delta \otimes \iota) \Delta
$$

and the cancellation properties

$$
[\Delta(A)(A \otimes 1)]=A \otimes A=[\Delta(A)(1 \otimes A)]
$$

If $\mathbb{G}=(A, \Delta)$ is a compact quantum group, there exists a unique state $h$ on $A$ which is invariant under the comultiplication:

$$
(\iota \otimes h) \Delta(a)=(h \otimes \iota) \Delta(a)=h(a) 1
$$

for all $a \in A$. We call $h$ the Haar state of $\mathbb{G}$.

Definition 1.3. Let $H$ be a Hilbert space. A unitary corepresentation $v$ of $\mathbb{G}$ on $H$ is a unitary element of $\mathrm{M}(\mathcal{K}(H) \otimes A)$ satisfying $(\iota \otimes \Delta)(v)=v_{12} v_{13}$. The dimension of the underlying Hilbert space $H$ is called the dimension of $v$ and denoted by $\operatorname{dim} v$.

The tensor product of the unitary corepresentations $v$ and $w$ is defined by

$$
v \odot w:=v_{13} w_{23}
$$

Note that the corepresentations $v \uparrow w$ and $w \uparrow v$ are in general not unitarily equivalent. This is a crucial feature of quantum groups. 
Notation 1.4. Let $\mathbb{G}=(A, \Delta)$ be a compact quantum group. Given two unitary corepresentations $v \in$ $\mathrm{M}(\mathcal{K}(H) \otimes A)$ and $w \in \mathrm{M}(\mathcal{K}(K) \otimes A)$, we denote by $\operatorname{Mor}(v, w)$ the intertwiners between $v$ and $w$ :

$$
\operatorname{Mor}(v, w)=\{S \in \mathrm{B}(H, K) \mid(S \otimes 1) v=w(S \otimes 1)\} .
$$

Terminology 1.5. A unitary corepresentation $v \in \mathrm{M}(\mathcal{K}(H) \otimes A)$ is called irreducible if $\operatorname{Mor}(v, v)=\mathbb{C} 1$. A unitary corepresentation $w \in \mathrm{M}(\mathcal{K}(L) \otimes A)$ is called unitarily equivalent to $v$ if $\operatorname{Mor}(v, w)$ contains a unitary operator.

In this paper, all corepresentations are assumed to be unitary. Recall the following well known facts (see [30]). Every irreducible corepresentation of a compact quantum group is finite dimensional and every corepresentation decomposes as a direct sum of irreducible corepresentations.

Notation 1.6. Let $\mathbb{G}=(A, \Delta)$ be a compact quantum group. We denote by $\widehat{\mathbb{G}}$ the set of equivalence classes of irreducible corepresentations of $\mathbb{G}$ and we choose unitary representatives $U^{x} \in \mathrm{B}\left(H_{x}\right) \otimes A$ for all $x \in \widehat{\mathbb{G}}$. We denote by $\epsilon$ the (class of the) trivial corepresentation $1 \in A$.

For every $x \in \widehat{\mathbb{G}}$, there is a unique $\bar{x} \in \widehat{\mathbb{G}}$ such that $\operatorname{Mor}(\epsilon, x \oplus \bar{x}) \neq 0$. The irreducible corepresentation $\bar{x}$ is called the adjoint of $x$. Take now $t \in \operatorname{Mor}(\epsilon, x \odot \bar{x}), t \neq 0$ and define the antilinear map

$$
S_{t}: H_{x} \rightarrow H_{\bar{x}}: \xi \mapsto\left(\xi^{*} \otimes 1\right) t .
$$

Define $Q_{x}:=S_{t}^{*} S_{t}$ and normalize $t$ in such a way that $\operatorname{Tr}\left(Q_{x}\right)=\operatorname{Tr}\left(Q_{x}^{-1}\right)$. This uniquely determines $Q_{x}$ and fixes $t$ up to a number of modulus 1 . If we take the unique $\tilde{t} \in \operatorname{Mor}(\epsilon, \bar{x} \odot x) \operatorname{such}$ that $\left(t^{*} \otimes 1\right)(1 \otimes \tilde{t})=1$, then $S_{\tilde{t}}=S_{t}^{-1}$ and $Q_{\bar{x}}=\left(S_{t} S_{t}^{*}\right)^{-1}$.

Notation 1.7. The quantum dimension of $x \in \widehat{\mathbb{G}}$ is defined as $\operatorname{Tr}\left(Q_{x}\right)$ and denoted by $\operatorname{dim}_{q}(x)$.

Observe that $t^{*} t=\operatorname{dim}_{q}(x) 1$ and that $\operatorname{dim}_{q}(\bar{x})=\operatorname{dim}_{q}(x) \geq \operatorname{dim}(x)$. The orthogonality relations can then be written as follows (see [30]): for $\xi \in H_{x}, \eta \in H_{y}$,

$$
(\iota \otimes h)\left(U^{x}\left(\xi \eta^{*} \otimes 1\right)\left(U^{y}\right)^{*}\right)=\frac{\delta_{x, y} 1}{\operatorname{dim}_{q}(x)}\left\langle Q_{x} \xi, \eta\right\rangle .
$$

Notation 1.8. Let $\mathbb{G}=(A, \Delta)$ be a compact quantum group. We denote by $\mathcal{A}$ the set of coefficients of finite dimensional corepresentations of $\mathbb{G}$. Hence,

$$
\mathcal{A}=\left\langle\left(\omega_{\xi, \eta} \otimes \iota\right)\left(U^{x}\right) \mid x \in \widehat{\mathbb{G}}, \xi, \eta \in H_{x}\right\rangle
$$

Then, $\mathcal{A}$ is a unital dense *-subalgebra of $A$. Restricting $\Delta$ to $\mathcal{A}, \mathcal{A}$ becomes a Hopf *-algebra.

Terminology 1.9. Let $\mathcal{B}$ be a unital *-algebra. A linear functional $\omega: \mathcal{B} \rightarrow \mathbb{C}$ is said to be a faithful state on $\mathcal{B}$ if $\omega(1)=1$ and if $\omega\left(a a^{*}\right) \geq 0$ for all $a \in \mathcal{B}$, with equality holding if and only if $a=0$.

Observe that the Haar state of a compact quantum group $(A, \Delta)$ is a faithful state on the underlying Hopf *-algebra $\mathcal{A}$.

The dual (discrete) quantum group of $(A, \Delta)$, can be defined as

$$
\hat{A}=\bigoplus_{x \in \widehat{\mathbb{G}}} \mathrm{B}\left(H_{x}\right) \quad \text { with } \quad \hat{\Delta}(a) S=S a \quad \text { for all } a \in \hat{A}, S \in \operatorname{Mor}(x, y \uparrow z) .
$$

There is, of course, another way to define $(\hat{A}, \hat{\Delta})$, using the left regular corepresentation of $(A, \Delta)$ and the theory of multiplicative unitaries. We only need this in Section 4, see Notation 4.6. 


\section{Spectral subspaces and quantum multiplicity}

In this section, we give a brief overview of the general theory of ergodic coactions of compact quantum groups. We study spectral subspaces and prove in particular that they are finite dimensional. The results in this section are well known (see [16] for the classical case of compact groups and [10, 18, 23] for compact quantum groups). We give a short presentation for the convenience of the reader.

Let $\mathbb{G}=(A, \Delta)$ be a compact quantum group. Recall that $\widehat{\mathbb{G}}$ denotes the set of equivalence classes of irreducible corepresentations of $\mathbb{G}$ and that we chose unitary representatives $U^{x} \in \mathrm{B}\left(H_{x}\right) \otimes A$ for all $x \in \widehat{\mathbb{G}}$. We have $(\iota \otimes \Delta)\left(U^{x}\right)=U_{12}^{x} U_{13}^{x}$.

Definition 2.1. Let $B$ be a unital $C^{*}$-algebra. A (right) coaction of $(A, \Delta)$ on $B$ is a unital *-homomorphism $\delta: B \rightarrow B \otimes A$ satisfying

$$
(\delta \otimes \iota) \delta=(\iota \otimes \Delta) \delta \quad \text { and } \quad[\delta(B)(1 \otimes A)]=B \otimes A
$$

The coaction $\delta$ is said to be ergodic if the fixed point algebra $B^{\delta}:=\{x \in A \mid \delta(x)=x \otimes 1\}$ equals $\mathbb{C} 1$.

Remark 2.2. If $\delta: B \rightarrow B \otimes A$ is an ergodic coaction of $(A, \Delta)$ on $B$ there is a unique state $\omega$ on $B$ which is invariant under $\delta$, given by $\omega(b) 1=(\iota \otimes h) \delta(b)$.

In what follows, we fix a coaction $\delta: B \rightarrow B \otimes A$ of a compact quantum group $\mathbb{G}=(A, \Delta)$.

Definition 2.3. Let $x \in \widehat{\mathbb{G}}$. We define the spectral subspace associated with $x$ by

$$
K_{x}=\left\{X \in \bar{H}_{x} \otimes B \mid(\iota \otimes \delta)(X)=X_{12} U_{13}^{x}\right\}
$$

Defining $\operatorname{Hom}\left(H_{x}, B\right)=\left\{S: H_{x} \rightarrow B \mid S\right.$ linear and $\left.\delta(S \xi)=(S \otimes \iota)\left(U^{x}(\xi \otimes 1)\right)\right\}$, we have $K_{x} \cong \operatorname{Hom}\left(H_{x}, B\right)$, associating to every $X \in K_{x}$ the operator $S_{X}: H_{x} \rightarrow B: \xi \mapsto X(\xi \otimes 1)$.

Definition 2.4. We define $\mathcal{B}$ as the subspace of $B$ generated by the spectral subspaces, i.e.

$$
\mathcal{B}:=\left\langle X(\xi \otimes 1) \mid x \in \widehat{\mathbb{G}}, X \in K_{x}, \xi \in H_{x}\right\rangle .
$$

Observe that $\mathcal{B}$ is a dense unital ${ }^{*}$-subalgebra of $B$ and that the restriction $\delta: \mathcal{B} \rightarrow \mathcal{B} \otimes \mathcal{A}$ defines a coaction of the Hopf *-algebra $(\mathcal{A}, \Delta)$ on $\mathcal{B}$.

Terminology 2.5. A coaction $\delta: B \rightarrow B \otimes A$ of $(A, \Delta)$ on $B$ is said to be universal if $B$ is the universal enveloping $\mathrm{C}^{*}$-algebra of $\mathcal{B}$. It is said to be reduced if the conditional expectation $(\iota \otimes h) \delta$ of $B$ on $B^{\delta}$ is faithful.

Remark 2.6. Observe that an ergodic coaction is reduced if and only if the unique invariant state is faithful.

In the special case where $B=A$ and $\delta=\Delta$, the ${ }^{*}$-algebra $\mathcal{B}$ coincides with the underlying Hopf ${ }^{*}$-algebra $\mathcal{A} \subset A$ consisting of coefficients of finite-dimensional corepresentations. So, we obtain the usual notions: a compact quantum group $(A, \Delta)$ is said to be universal if $A$ is the universal enveloping $\mathrm{C}^{*}$-algebra of $\mathcal{A}$ and reduced if the Haar state is faithful on $A$. Clearly, any compact quantum group has its universal and reduced companion. In the case where $(A, \Delta)$ is the dual of a discrete group, these notions coincide with the full, resp. reduced group $C^{*}$-algebra.

A compact quantum group is said to be co-amenable its universal and reduced companion coincide. Equivalently, a compact quantum group is co-amenable if we have on the same $\mathrm{C}^{*}$-algebra a bounded co-unit and a faithful Haar state. It is then clear that a coaction of a co-amenable compact quantum group is always both universal and reduced. Examples of co-amenable compact quantum groups include $\mathrm{SU}_{q}(n)$ and other $q$-deformations of compact Lie groups.

Fix now an ergodic coaction $\delta: B \rightarrow B \otimes A$ of a compact quantum group $(A, \Delta)$ on a unital $\mathrm{C}^{*}$-algebra $B$. Denote by $\omega$ the unique invariant state on $B$.

For every $X, Y \in K_{x}, X Y^{*} \in B^{\delta}=\mathbb{C} 1$. So, for $x \in \widehat{\mathbb{G}}$, the spectral subspace $K_{x}$ is a Hilbert space with scalar product $\langle X, Y\rangle 1=X Y^{*}$. 
Terminology 2.7. Let $\delta$ be an ergodic coaction of $(A, \Delta)$ on $B$. The dimension of the Hilbert space $K_{x}$ is called the multiplicity of $x$ in $\delta$ and denoted by $\operatorname{mult}(x)$.

Define, for every $x \in \widehat{\mathbb{G}}$, the element $X^{x} \in \mathrm{B}\left(H_{x}, \bar{K}_{x}\right) \otimes B$ such that $\left(X^{x}\right)^{*}(\bar{Y} \otimes 1)=Y^{*}$ for all $Y \in K_{x}$. Observe that $X^{x}\left(X^{x}\right)^{*}=1$. Therefore, $\left(X^{x}\right)^{*} X^{x} \in \mathrm{B}\left(H_{x}\right) \otimes B$ is a projection.

Let $x \in \widehat{\mathbb{G}}$. Take $t \in \operatorname{Mor}(\epsilon, \bar{x} \odot x)$, normalized in such a way that $t^{*} t=\operatorname{dim}_{q}(x)$. Define the antilinear map

$$
T_{t}: K_{x} \rightarrow K_{\bar{x}}: T_{t}(Y)=\left(t^{*} \otimes 1\right)\left(1 \otimes Y^{*}\right) .
$$

Since $t$ is fixed up to a number of modulus one, $L_{x}:=T_{t}^{*} T_{t}$ is a well defined positive element of $\mathrm{B}\left(K_{x}\right)$.

Definition 2.8. We put $\operatorname{mult}_{q}(x):=\sqrt{\operatorname{Tr}\left(L_{x}\right) \operatorname{Tr}\left(L_{\bar{x}}\right)}$ and call $\operatorname{mult}_{q}(x)$ the quantum multiplicity of $x$ in $\delta$. We prove in Theorem 2.9 that $\operatorname{mult}_{q}(x) \leq \operatorname{dim}_{q}(x)$ for all $x \in \widehat{\mathbb{G}}$. If equality holds for all $x \in \widehat{\mathbb{G}}$, we say that $\delta$ is of full quantum multiplicity.

Theorem 2.9. Let $\delta: B \rightarrow B \otimes A$ be an ergodic coaction of a compact quantum group $\mathbb{G}=(A, \Delta)$ on a unital $C^{*}$-algebra $B$. For every irreducible corepresentation $x \in \widehat{\mathbb{G}}$,

$$
\operatorname{mult}(x) \leq \operatorname{mult}_{q}(x) \leq \operatorname{dim}_{q}(x) .
$$

With $X^{x}$ defined as above, the ergodic coaction is of full quantum multiplicity if and only if $X^{x}$ is unitary for all $x \in \widehat{\mathbb{G}}$.

Proof. Let $x \in \widehat{\mathbb{G}}$ and take $t \in \operatorname{Mor}(\epsilon, \bar{x} \odot x)$, normalized in such a way that $t^{*} t=\operatorname{dim}_{q}(x)$. If we take the unique $\tilde{t} \in \operatorname{Mor}(\epsilon, x \Theta \bar{x})$ satisfying $\left(1 \otimes t^{*}\right)(\tilde{t} \otimes 1)=1$, we have $\tilde{t}^{*} \tilde{t}=\operatorname{dim}_{q}(\bar{x})$ and hence, it is clear that $T_{\tilde{t}}=T_{t}^{-1}$ and $L_{\bar{x}}=\left(T_{t} T_{t}^{*}\right)^{-1}$. So,

$$
\operatorname{mult}_{q}(x)=\sqrt{\operatorname{Tr}\left(T_{t} T_{t}^{*}\right) \operatorname{Tr}\left(\left(T_{t} T_{t}^{*}\right)^{-1}\right)} \geq \operatorname{dim}\left(T_{t}\right)=\operatorname{mult}(x),
$$

for all $x \in \widehat{\mathbb{G}}$. By definition

$$
\left\langle L_{x} X, Y\right\rangle 1=\left\langle\left(t^{*} \otimes 1\right)\left(1 \otimes Y^{*}\right),\left(t^{*} \otimes 1\right)\left(1 \otimes X^{*}\right)\right\rangle 1=\left(t^{*} \otimes 1\right)\left(1 \otimes Y^{*} X\right)(t \otimes 1) .
$$

Since $t^{*}(1 \otimes a) t=\operatorname{Tr}\left(Q_{x}^{-1} a\right)$ for all $a \in \mathrm{B}\left(H_{x}\right)$, we conclude that

$$
\operatorname{Tr}\left(L_{x}\right) 1=\left(\operatorname{Tr}\left(Q_{x}^{-1} \cdot\right) \otimes \iota\right)\left(\left(X^{x}\right)^{*} X^{x}\right) .
$$

Since $\left(X^{x}\right)^{*} X^{*}$ is a projection, it follows that $\operatorname{Tr}\left(L_{x}\right) \leq \operatorname{Tr}\left(Q_{x}^{-1}\right)=\operatorname{dim}_{q}(x)$ for all $x \in \widehat{\mathbb{G}}$. Applying this inequality to $x$ and $\bar{x}$, we conclude that $\operatorname{mult}_{q}(x) \leq \operatorname{dim}_{q}(x)$ for all $x \in \widehat{\mathbb{G}}$.

Moreover, $\operatorname{mult}_{q}(x)=\operatorname{dim}_{q}(x)$ for all $x \in \widehat{\mathbb{G}}$ if and only if $\left(\operatorname{Tr}\left(Q_{x}^{-1} \cdot\right) \otimes \iota\right)\left(\left(X^{x}\right)^{*} X^{x}\right)=\operatorname{Tr}\left(Q_{x}^{-1}\right) 1$ for all $x \in \widehat{\mathbb{G}}$. This last statement holds if and only if $\left(X^{x}\right)^{*} X^{x}=1$ for all $x \in \widehat{\mathbb{G}}$, i.e. when $X^{x}$ is unitary for all $x \in \widehat{\mathbb{G}}$.

It is also straightforward to show that the invariant state $\omega$ is a KMS state, at least for universal and for reduced ergodic coactions.

Proposition 2.10. If the ergodic coaction $\delta$ of $(A, \Delta)$ on $B$ is either universal or reduced, the invariant state $\omega$ is a KMS state. The elements of the dense ${ }^{*}$-subalgebra $\mathcal{B} \subset B$ (Definition 2.4) are analytic with respect to the modular group, given by

$$
\sigma_{t}^{\omega}(Y(\xi \otimes 1))=\left(L_{x}^{-i t} Y\right)\left(Q_{x}^{i t} \xi \otimes 1\right) \quad \text { for all } x \in \widehat{\mathbb{G}}, Y \in K_{x}, \xi \in H_{x} .
$$

Proof. Let $y, z \in \widehat{\mathbb{G}}$ and $Y \in K_{y}, Z \in K_{z}$. One verifies that $\left(U^{z}\right)^{*}\left((\iota \otimes \omega)\left(Z^{*} Y\right) \otimes 1\right) U^{y}=(\iota \otimes \omega)\left(Z^{*} Y\right) \otimes 1$. Hence, $(\iota \otimes \omega)\left(Z^{*} Y\right)$ equals 0 if $y \neq z$ and is scalar if $y=z$. Applying $\omega$ to (2.1), we conclude that

$$
(\iota \otimes \omega)\left(Z^{*} Y\right)=\frac{\delta_{y, z} 1}{\operatorname{dim}_{q}(y)}\left\langle L_{y} Y, Z\right\rangle .
$$


If moreover $\xi \in H_{y}, \eta \in H_{z}$ and if we put $a=Y(\xi \otimes 1)$ and $b=Z(\eta \otimes 1)$, we get

$$
\omega\left(b^{*} a\right)=\frac{\delta_{y, z}}{\operatorname{dim}_{q}(y)}\langle\xi, \eta\rangle\left\langle L_{y} Y, Z\right\rangle .
$$

Using (1.1), one checks that

$$
\omega\left(a b^{*}\right)=(\iota \otimes \omega)\left(Y\left(\xi \eta^{*} \otimes 1\right) Z^{*}\right)=\frac{\delta_{y, z} 1}{\operatorname{dim}_{q}(y)}\left\langle Q_{y} \xi, \eta\right\rangle\langle Y, Z\rangle .
$$

As a linear space, $\mathcal{B} \cong \bigoplus_{x \in \widehat{\mathbb{G}}}\left(K_{x} \otimes H_{x}\right)$. So, we can define linear maps $\sigma_{t}^{\omega}: \mathcal{B} \rightarrow \mathcal{B}$ by the formula

$$
\sigma_{t}^{\omega}(Y(\xi \otimes 1)):=L_{y}^{-i t}(Y)\left(Q_{y}^{i t} \xi \otimes 1\right) .
$$

It is clear that $\left(\sigma_{t}^{\omega}\right)$ is a one-parameter group of linear isomorphisms of $\mathcal{B}$. Observe that all elements of $\mathcal{B}$ are analytic with respect to $\left(\sigma_{t}^{\omega}\right)$ and that $\omega\left(\sigma_{i}^{\omega}(a) b^{*}\right)=\omega\left(b^{*} a\right)=\omega\left(a \sigma_{i}^{\omega}(b)^{*}\right)$ for all $a, b \in \mathcal{B}$. Since $(2.2)$ implies that $\omega$ is faithful on $\mathcal{B}$, it follows that $\sigma_{i}^{\omega}: \mathcal{B} \rightarrow \mathcal{B}$ is multiplicative and that $\sigma_{i}^{\omega}(a)^{*}=\sigma_{-i}^{\omega}\left(a^{*}\right)$ for all $a \in \mathcal{B}$. Standard complex analysis allows to conclude that the $\sigma_{t}^{\omega}$ are ${ }^{*}$-automorphisms of $\mathcal{B}$. It is also clear that $\omega$ is invariant under $\sigma_{t}^{\omega}$.

If $\delta$ is a universal coaction, the one-parameter group $\left(\sigma_{t}^{\omega}\right)$ extends to $B$ by universality. If $\delta$ is a reduced coaction, we can extend $\sigma_{t}^{\omega}$ to $B$ because $\omega$ is invariant under $\sigma_{t}^{\omega}$ and $\omega$ is faithful on $B$. In both cases, it follows that $\left(\sigma_{t}^{\omega}\right)$ satisfies the KMS condition with respect to $\omega$ and so, $\omega$ is a KMS state.

Finally observe that $\omega$ is a trace if and only if $L_{x}=1$ and $Q_{x}=1$ for all $x \in \mathbb{G}$ with $K_{x} \neq 0$.

\section{Commuting coactions and monoidal equivalence of quantum groups}

Our main goal in this section is to show the relation between ergodic coactions of full quantum multiplicity and monoidal equivalence of compact quantum groups. In Sections 5 and 6 , we shall give examples of monoidally equivalent compact quantum groups, giving rise to new examples of ergodic coactions.

The relation between ergodic coactions of full quantum multiplicity and monoidal equivalence allows us to classify completely such coactions for the unitary and orthogonal quantum groups $A_{u}(F)$ and $A_{o}(F)$, in particular for $\mathrm{SU}_{q}(2)$.

Definition 3.1. Two compact quantum groups $\mathbb{G}=(A, \Delta)$ and $\mathbb{G}_{2}=\left(A_{2}, \Delta_{2}\right)$ are said to be monoidally equivalent if there exists a bijection $\varphi: \widehat{\mathbb{G}} \rightarrow \widehat{\mathbb{G}}_{2}$ satisfying $\varphi(\epsilon)=\epsilon$, together with linear isomorphisms

$$
\varphi: \operatorname{Mor}\left(x_{1} \uparrow \cdots \uparrow x_{r}, y_{1} \uparrow \cdots \oplus y_{k}\right) \rightarrow \operatorname{Mor}\left(\varphi\left(x_{1}\right) \uparrow \cdots \oplus \varphi\left(x_{r}\right), \varphi\left(y_{1}\right) \uparrow \cdots \oplus \varphi\left(y_{k}\right)\right)
$$

satisfying the following conditions:

$$
\begin{aligned}
\varphi(1) & =1 & \varphi(S \otimes T) & =\varphi(S) \otimes \varphi(T) \\
\varphi\left(S^{*}\right) & =\varphi(S)^{*} & \varphi(S T) & =\varphi(S) \varphi(T)
\end{aligned}
$$

whenever the formulas make sense. In the first formula, we consider $1 \in \operatorname{Mor}(x, x)=\operatorname{Mor}(x, x \odot \epsilon)=$ $\operatorname{Mor}(x, \epsilon \uparrow x)$. Such a collection of maps $\varphi$ is called a monoidal equivalence between $\mathbb{G}$ and $\mathbb{G}_{2}$.

Remark 3.2. To define a monoidal equivalence between $\mathbb{G}$ and $\mathbb{G}_{2}$, it suffices to define a bijection $\varphi: \widehat{\mathbb{G}} \rightarrow \widehat{\mathbb{G}}_{2}$ satisfying $\varphi(\epsilon)=\epsilon$, together with linear isomorphisms $\varphi: \operatorname{Mor}\left(x, y_{1}(\top \cdots) y_{k}\right) \rightarrow \operatorname{Mor}\left(\varphi(x), \varphi\left(y_{1}\right)\right.$ (T) $\cdots$ (T) $\left.\varphi\left(y_{k}\right)\right)$ for $k=1,2,3$, satisfying

$$
\begin{aligned}
& \varphi(1)=1 \\
& \varphi(S)^{*} \varphi(T)=\varphi\left(S^{*} T\right) \\
& \text { for all } S \in \operatorname{Mor}(a, x \uparrow y), T \in \operatorname{Mor}(b, x \Subset y) \\
& \varphi((S \otimes 1) T)=(\varphi(S) \otimes 1) \varphi(T) \quad \text { for all } \quad T \in \operatorname{Mor}(a, b \odot z), S \in \operatorname{Mor}(b, x \odot y) \\
& \varphi((1 \otimes S) T)=(1 \otimes \varphi(S)) \varphi(T) \quad \text { for all } \quad T \in \operatorname{Mor}(a, x \circledast b), S \in \operatorname{Mor}(b, y \circledast z)
\end{aligned}
$$


Indeed, such a $\varphi$ admits a unique extension to a monoidal equivalence. Again, (3.2) should be valid for $1 \in \operatorname{Mor}(x, x)=\operatorname{Mor}(x, x \oplus \epsilon)=\operatorname{Mor}(x, \epsilon \top x)$.

Remark 3.3. Observe that the existence of the linear isomorphisms $\varphi: \operatorname{Mor}(a, b \Subset c) \rightarrow \operatorname{Mor}(\varphi(a), \varphi(b) \oplus$ $\varphi(c)$ ) only says that $\mathbb{G}$ and $\mathbb{G}_{2}$ have the same fusion rules. Adding (3.2)-(3.5) means that $\mathbb{G}$ and $\mathbb{G}_{2}$ moreover have the same $6 j$-symbols (see [12]). Indeed, taking orthonormal bases for all $\operatorname{Mor}(a, b \odot c)$, we can write two natural orthonormal bases for $\operatorname{Mor}(a, x \odot y \uparrow z)$, one given by elements $(S \otimes 1) T$, the other given by elements $(1 \otimes S) T$. The coefficients of the transition unitary between both orthonormal bases are called the $6 j$-symbols of $\mathbb{G}$.

Remark 3.4. As we shall see in Sections 5 and 6, there are natural examples of monoidal equivalences where $\operatorname{dim}(\varphi(x)) \neq \operatorname{dim}(x)$. On the other hand, it is clear that $\operatorname{dim}_{q}(\varphi(x))=\operatorname{dim}_{q}(x)$ for all $x \in \mathbb{G}$ and all monoidal equivalences $\varphi$. We shall see in Section 6 that for a certain class of compact quantum groups (the universal unitary ones), this equality of quantum dimension is the only constraint for monoidal equivalence.

Remark 3.5. It is clear that a monoidal equivalence $\varphi$ in the sense of Definition 3.1, defines a monoidal equivalence in the usual sense (preserving the *-operation), between the monoidal categories of finite dimensional corepresentations of $\mathbb{G}$ and $\mathbb{G}_{2}([20])$. Moreover, this monoidal equivalence is uniquely determined up to isomorphism. We prefer to work with the 'concrete' data of Definition 3.1, avoiding all kinds of identifications.

Notation 3.6. If two compact quantum groups $\mathbb{G}=(A, \Delta)$ and $\mathbb{G}_{2}=\left(A_{2}, \Delta_{2}\right)$ are monoidally equivalent, we write $\mathbb{G} \underset{\text { mon }}{\sim} \mathbb{G}_{2}$

Closely related to the notion of monoidal equivalence, is the following notion of unitary fiber functor (see Proposition 3.12 for the relation between both notions).

Definition 3.7. Let $\mathbb{G}=(A, \Delta)$ be a compact quantum group. A unitary fiber functor associates to every $x \in \widehat{\mathbb{G}}$ a finite dimensional Hilbert space $H_{\varphi(x)}$ and consists further of linear maps

$$
\varphi: \operatorname{Mor}\left(x_{1} \uparrow \cdots \Subset x_{r}, y_{1} \uparrow \cdots \Subset y_{k}\right) \rightarrow \mathrm{B}\left(H_{\varphi\left(x_{1}\right)} \otimes \cdots \otimes H_{\varphi\left(x_{r}\right)}, H_{\varphi\left(y_{1}\right)} \otimes \cdots \otimes H_{\varphi\left(y_{k}\right)}\right)
$$

satisfying equations (3.1) in Definition 3.1.

Remark 3.8. We make a remark analogous to 3.2. To define a unitary fiber functor on $\mathbb{G}$, it suffices to associate to every $x \in \widehat{\mathbb{G}}$ a finite-dimensional Hilbert space $H_{\varphi(x)}$, with $H_{\varphi(\epsilon)}=\mathbb{C}$ and to define linear maps $\varphi: \operatorname{Mor}\left(x, y_{1}\left(\cdots \uparrow y_{k}\right) \rightarrow \mathrm{B}\left(H_{\varphi(x)}, H_{\varphi\left(y_{1}\right)} \otimes \cdots \otimes H_{\varphi\left(y_{k}\right)}\right)\right.$ for $k=1,2,3$, satisfying (3.2) - (3.5) as well as the non-degenerateness assumption

$$
\left\{\varphi(S) \xi \mid a \in \widehat{\mathbb{G}}, S \in \operatorname{Mor}(a, b \Subset c), \xi \in H_{\varphi(a)}\right\} \quad \text { is total in } \quad H_{\varphi(b)} \otimes H_{\varphi(c)}
$$

for all $b, c \in \widehat{\mathbb{G}}$.

Moreover, it follows from Proposition 3.12 below, that a unitary fiber functor $\varphi$ on $\mathbb{G}$ naturally defines a compact quantum group $\mathbb{G}_{2}$ such that $\varphi$ becomes a monoidal equivalence between $\mathbb{G}$ and $\mathbb{G}_{2}$.

Theorem 3.9. Consider a compact quantum group $\mathbb{G}=(A, \Delta)$ and let $\varphi$ be a unitary fiber functor on $\mathbb{G}$.

- There exists a unique unital ${ }^{*}$-algebra $\mathcal{B}$ equipped with a faithful state $\omega$ and unitary elements $X^{x} \in$ $\mathrm{B}\left(H_{x}, H_{\varphi(x)}\right) \otimes \mathcal{B}$ for all $x \in \widehat{\mathbb{G}}$, satisfying

1. $X_{13}^{y} X_{23}^{z}(S \otimes 1)=(\varphi(S) \otimes 1) X^{x} \quad$ for all $\quad S \in \operatorname{Mor}(x, y \oplus z)$,

2. the matrix coefficients of the $X^{x}$ form a linear basis of $\mathcal{B}$,

3. $(\iota \otimes \omega)\left(X^{x}\right)=0 \quad$ if $\quad x \neq \epsilon$.

- There exists a unique coaction $\delta: \mathcal{B} \rightarrow \mathcal{B} \otimes \mathcal{A}$ satisfying

$$
(\iota \otimes \delta)\left(X^{x}\right)=X_{12}^{x} U_{13}^{x}
$$

for all $x \in \widehat{\mathbb{G}}$. 
- The state $\omega$ is invariant under $\delta$. Denoting by $B_{r}$ the $C^{*}$-algebra generated by $\mathcal{B}$ in the GNSrepresentation associated with $\omega$ and denoting by $B_{u}$ the universal enveloping $C^{*}$-algebra of $\mathcal{B}$, the coaction $\delta$ admits a unique extension to a coaction on $B_{r}$, resp. $B_{u}$.

These coactions are reduced, resp. universal and they are ergodic and of full quantum multiplicity.

- Every reduced, resp. universal, ergodic coaction of full quantum multiplicity, arises in this way from a unitary fiber functor.

Proof. Let $\varphi$ be a unitary fiber functor on $\mathbb{G}=(A, \Delta)$. Define the vector space $\mathcal{B}=\oplus_{x \in \widehat{\mathbb{G}}} \mathrm{B}\left(H_{x}, H_{\varphi(x)}\right)^{*}$. We shall turn this vector space into a ${ }^{*}$-algebra.

Define natural elements $X^{x} \in \mathrm{B}\left(H_{x}, H_{\varphi(x)}\right) \otimes \mathcal{B}$ by $\left(\omega_{x} \otimes \iota\right)\left(X^{x}\right)=\left(\delta_{x, y} \omega_{x}\right)_{y \in \widehat{\mathbb{G}}}$ for all $\omega_{x} \in \mathrm{B}\left(H_{x}, H_{\varphi(x)}\right)^{*}$. By definition, the coefficients of the $X^{x}$ form a linear basis of $\mathcal{B}$. Hence, it suffices to define a product and an involution on the level of the $X^{x}$.

It is clear that there exists a unique bilinear multiplication map $\mathcal{B} \times \mathcal{B} \rightarrow \mathcal{B}$ such that

$$
X_{13}^{y} X_{23}^{z}(S \otimes 1)=(\varphi(S) \otimes 1) X^{x} \quad \text { for all } \quad S \in \operatorname{Mor}(x, y \Subset z) .
$$

But then

$\left(X_{14}^{a} X_{24}^{b}\right) X_{34}^{c}((S \otimes 1) T \otimes 1)=(\varphi(S) \otimes 1 \otimes 1) X_{13}^{y} X_{23}^{c}(T \otimes 1)=((\varphi(S) \otimes 1) \varphi(T) \otimes 1) X^{x}=(\varphi((S \otimes 1) T) \otimes 1) X^{x}$ for all $S \in \operatorname{Mor}(y, a \circledast b), T \in \operatorname{Mor}(x, y \circledast c)$. Since intertwiners of the form $(S \otimes 1) T$ linearly span $\operatorname{Mor}(x, a \oplus$ $b \odot c)$, we conclude that

$$
\left(X_{14}^{a} X_{24}^{b}\right) X_{34}^{c}(S \otimes 1)=(\varphi(S) \otimes 1) X^{x}
$$

for all $S \in \operatorname{Mor}(x, a \uparrow b \uparrow c)$. Analogously,

$$
X_{14}^{a}\left(X_{24}^{b} X_{34}^{c}\right)(S \otimes 1)=(\varphi(S) \otimes 1) X^{x}
$$

for all $S \in \operatorname{Mor}(x, a \oplus b \oplus c)$. This proves the associativity of the product on $\mathcal{B}$. It is clear that $X^{\epsilon}$ provides the unit element of $\mathcal{B}$.

Observe also that

$$
\left(\varphi(S)^{*} \otimes 1\right) X_{13}^{x} X_{23}^{y}=X^{z}\left(S^{*} \otimes 1\right)
$$

for all $S \in \operatorname{Mor}(z, x \odot y)$.

We define an antilinear map $b \mapsto b^{*}$ on $\mathcal{B}$ such that

$$
\left(X^{x}\right)_{13}^{*}(\varphi(t) \otimes 1)=X_{23}^{\bar{x}}(t \otimes 1)
$$

for all $x \in \widehat{\mathbb{G}}, t \in \operatorname{Mor}(\epsilon, x \uparrow \bar{x})$. This antilinear map is well defined: taking $t \in \operatorname{Mor}(\epsilon, x \uparrow \bar{x})$ and $\tilde{t} \in \operatorname{Mor}(\epsilon, \bar{x} \odot x)$, normalized in such a way that $\left(t^{*} \otimes 1\right)(1 \otimes \tilde{t})=1$, we define

$$
\left(\left(\omega_{\xi, \eta} \otimes \iota\right)\left(X^{x}\right)\right)^{*}:=\left(\omega_{\left(\xi^{*} \otimes 1\right) t,\left(1 \otimes \eta^{*}\right) \varphi(\tilde{t})} \otimes \iota\right)\left(X^{\bar{x}}\right)
$$

for all $\xi \in H_{x}$ and $\eta \in H_{\varphi(x)}$.

For $\xi \in H_{x}, \eta \in H_{\varphi(x)}$, we compute

$$
\begin{aligned}
\left(\left(\left(\omega_{\xi, \eta} \otimes \iota\right)\left(X^{x}\right)\right)^{*}\right)^{*} & =\left(\left(\omega_{\left(\xi^{*} \otimes 1\right) t,\left(1 \otimes \eta^{*}\right) \varphi(\tilde{t})} \otimes \iota\right)\left(X^{\bar{x}}\right)\right)^{*} \\
& =\left(\omega_{\left(\left(\left(\xi^{*} \otimes 1\right) t\right)^{*} \otimes 1\right) \tilde{t},\left(1 \otimes\left(\left(1 \otimes \eta^{*}\right) \varphi(\tilde{t})\right)^{*} \varphi(t)\right)} \otimes \iota\right)\left(X^{x}\right) \\
& =\left(\omega_{\xi, \eta} \otimes \iota\right)\left(X^{x}\right),
\end{aligned}
$$

in the last step using our particular choice of $t$ and $\tilde{t}$.

We also get

$$
\left(t^{*} \otimes 1\right)\left(X_{23}^{\bar{x}}\right)^{*}=\left(\varphi(t)^{*} \otimes 1\right) X_{13}^{x} .
$$


Because

$$
\left(X^{x}\left(X^{x}\right)^{*}\right)_{13}(\varphi(t) \otimes 1)=X_{13}^{x} X_{23}^{\bar{x}}(t \otimes 1)=\varphi(t) \otimes 1
$$

and because, by (3.6),

$$
\left(t^{*} \otimes 1\right)\left(\left(X^{\bar{x}}\right)^{*} X^{\bar{x}}\right)_{23}=t^{*} \otimes 1
$$

the elements $X^{x}$ are unitaries.

Since for all $x, y, z \in \widehat{\mathbb{G}}$ and $S \in \operatorname{Mor}(z, x \oplus y)$,

$$
\left(X_{13}^{x} X_{23}^{y}(S \otimes 1)\right)^{*}=\left((\varphi(S) \otimes 1) X^{z}\right)^{*}=\left(X^{z}\right)^{*}\left(\varphi(S)^{*} \otimes 1\right)=\left(S^{*} \otimes 1\right)\left(X_{23}^{y}\right)^{*}\left(X_{13}^{x}\right)^{*}
$$

by (3.6) and the fact that the $X^{x}$ are unitary, our involution is anti-multiplicative. We conclude that $\mathcal{B}$ a *-algebra.

Denote by $\omega$ the linear functional $\omega: \mathcal{B} \rightarrow \mathbb{C}$ given by $\omega(1)=1$ and $(\iota \otimes \omega)\left(X^{x}\right)=0$ for all $x \neq \epsilon$. We show that $\omega$ is a faithful state on $\mathcal{B}$.

Let $x, y \in \widehat{\mathbb{G}}$. Take $t \in \operatorname{Mor}(\epsilon, x \odot \bar{x})$ such that $t^{*} t=\operatorname{dim}_{q}(x)$. Take $\tilde{t} \in \operatorname{Mor}(\epsilon, \bar{x} \odot x)$ such that $\left(t^{*} \otimes 1\right)(1 \otimes \tilde{t})=$ 1. Then,

$$
\left(\omega_{\mu, \rho} \otimes \iota\right)\left(X^{y}\right)\left(\omega_{\xi, \eta} \otimes \iota\right)\left(X^{x}\right)^{*}=\left(\omega_{\mu \otimes\left(\xi^{*} \otimes 1\right) t, \rho \otimes\left(1 \otimes \eta^{*}\right) \varphi(\tilde{t})} \otimes \iota\right)\left(X_{13}^{y} X_{23}^{\bar{x}}\right) .
$$

We conclude that

$$
\begin{aligned}
\omega\left(\left(\omega_{\mu, \rho} \otimes \iota\right)\left(X^{y}\right)\left(\omega_{\xi, \eta} \otimes \iota\right)\left(X^{x}\right)^{*}\right) & =\delta_{x, y} \frac{1}{\operatorname{dim}_{q}(x)}\left\langle\mu \otimes\left(\xi^{*} \otimes 1\right) t, t\right\rangle\left\langle\varphi(t), \rho \otimes\left(1 \otimes \eta^{*}\right) \varphi(\tilde{t})\right\rangle \\
& =\delta_{x, y} \frac{1}{\operatorname{dim}_{q}(x)}\left\langle\left(\xi^{*} \otimes 1\right) t,\left(\mu^{*} \otimes 1\right) t\right\rangle\langle\rho, \eta\rangle \\
& =\delta_{x, y} \frac{1}{\operatorname{dim}_{q}(x)}\left\langle Q_{x} \mu, \xi\right\rangle\langle\rho, \eta\rangle .
\end{aligned}
$$

Choose orthonormal bases $\left(f_{i}^{x}\right)$ for every space $H_{\varphi(x)}$. Any element $a \in \mathcal{B}$ admits a unique decomposition

$$
a=\sum_{x, i}\left(\omega_{\xi_{i}^{x}, f_{i}^{x}} \otimes \iota\right)\left(X^{x}\right)
$$

in terms of vectors $\xi_{i}^{x} \in H_{x}$. Then,

$$
\omega\left(a a^{*}\right)=\sum_{x, i} \frac{1}{\operatorname{dim}_{q}(x)}\left\langle Q_{x} \xi_{i}^{x}, \xi_{i}^{x}\right\rangle .
$$

It follows that $\omega\left(a a^{*}\right) \geq 0$ for all $a$ and that $\omega\left(a a^{*}\right)=0$ if and only if $\xi_{i}^{x}=0$ for all $x$ and $i$, i.e. if and only if $a=0$.

The definition of the coaction $\delta: \mathcal{B} \rightarrow \mathcal{B} \otimes \mathcal{A}$ is obvious and it is clear that $\omega$ is invariant under $\delta$. It follows that we can extend $\delta$ to coactions $\delta_{r}$, resp. $\delta_{u}$ of $(A, \Delta)$ on $B_{r}$, resp. $B_{u}$. Moreover, $\omega(x) 1=(\iota \otimes h) \delta_{r}(x)$ for all $x \in B_{r}$ and analogously for $x \in B_{u}$. It follows that $\delta_{r}$ and $\delta_{u}$ are ergodic coactions. Given the unitary elements $X^{x}$ and Theorem 2.9, it follows that $\delta_{r}$ and $\delta_{u}$ are of full quantum multiplicity.

By definition, the coaction $\delta_{r}$ on $B_{r}$ is reduced and the coaction $\delta_{u}$ on $B_{u}$ is universal. Indeed, the canonical *-subalgebra of $B_{u}$ generated by the spectral subspaces for $\delta_{u}$, is exactly $\mathcal{B}$.

It remains to show that any reduced, resp. universal ergodic coaction of full quantum multiplicity arises as above from a unitary fiber functor. Let $\delta: B \rightarrow B \otimes A$ be an ergodic coaction of full quantum multiplicity. Construct for $x \in \widehat{\mathbb{G}}$, the unitary elements $X^{x} \in \mathrm{B}\left(H_{x}, \bar{K}_{x}\right) \otimes B$ as in Section 2. Define $H_{\varphi(x)}:=\bar{K}_{x}$. Let $S \in \operatorname{Mor}\left(x_{1} \uparrow \cdots \top x_{r}, y_{1} \uparrow \cdots \top y_{k}\right)$. The element

$$
X_{1, k+1}^{y_{1}} \cdots X_{k, k+1}^{y_{k}}(S \otimes 1)\left(X_{1, r+1}^{x_{1}} \cdots X_{r, r+1}^{x_{r}}\right)^{*}
$$

is invariant under $\iota \otimes \delta$. So, we can define $\varphi(S)$ by the formula

$$
X_{1, k+1}^{y_{1}} \cdots X_{k, k+1}^{y_{k}}(S \otimes 1)=(\varphi(S) \otimes 1) X_{1, r+1}^{x_{1}} \cdots X_{r, r+1}^{x_{r}}
$$


for all $S \in \operatorname{Mor}\left(x_{1} \uparrow \cdots \oplus x_{r}, y_{1} \uparrow \cdots \uparrow y_{k}\right)$. It is clear that $\varphi$ is a unitary fiber functor on $\mathbb{G}$.

Denote by $\mathcal{B}$ the ${ }^{*}$-subalgebra of $B$ generated by the spectral subspaces of $\delta$ as defined in Section 2 . By definition, $\mathcal{B}$ is generated by the coefficients of the $X^{x}$. In order to show that $\mathcal{B}$ is isomorphic to the *-algebra defined by the unitary fiber functor $\varphi$, it suffices to show that the coefficients of the $X^{x}$ form a linear basis of $\mathcal{B}$. But this follows immediately from (2.2).

Definition 3.10. Two unitary fiber functors $\varphi$ and $\psi$ on a compact quantum group $\mathbb{G}$ are said to be isomorphic if there exist unitaries $u_{x} \in \mathrm{B}\left(H_{\varphi(x)}, H_{\psi(x)}\right)$ satisfying

$$
\psi(S)=\left(u_{y_{1}} \otimes \cdots \otimes u_{y_{k}}\right) \varphi(S)\left(u_{x_{1}}^{*} \otimes \cdots \otimes u_{x_{r}}^{*}\right)
$$

for all $S \in \operatorname{Mor}\left(x_{1} \uparrow \cdots \odot x_{r}, y_{1} \uparrow \cdots \odot y_{k}\right)$.

Proposition 3.11. Let $\varphi$ and $\psi$ be unitary fiber functors on $\mathbb{G}$ and denote by $\delta_{\varphi}$, resp. $\delta_{\psi}$ the associated coactions on $\mathcal{B}_{\varphi}$, resp. $\mathcal{B}_{\psi}$. Then the following statements are equivalent.

- The fiber functors $\varphi$ and $\psi$ are isomorphic.

- There exists $a^{*}$-isomorphism $\pi: \mathcal{B}_{\varphi} \rightarrow \mathcal{B}_{\psi}$ satisfying $(\pi \otimes \iota) \delta_{\varphi}=\delta_{\psi} \pi$.

Proof. Straightforward.

The following proposition follows immediately from the Tannaka-Krein reconstruction theorem, but we give a detailed statement for clarity. Its proof is completely analogous to the proof of Theorem 3.9.

Proposition 3.12. Let $\varphi$ be a unitary fiber functor on a compact quantum group $\mathbb{G}=(A, \Delta)$.

- There exists a unique universal compact quantum group $\left(A_{2}, \Delta_{2}\right)$ with underlying Hopf ${ }^{*}$-algebra $\left(\mathcal{A}_{2}, \Delta_{2}\right)$ and unitary corepresentations $U^{\varphi(x)} \in \mathrm{B}\left(H_{\varphi(x)}\right) \otimes \mathcal{A}_{2}$ satisfying

1. $U_{13}^{\varphi(y)} U_{23}^{\varphi(z)}(\varphi(S) \otimes 1)=(\varphi(S) \otimes 1) U^{\varphi(x)} \quad$ for all $S \in \operatorname{Mor}(x, y \circledast z)$,

2. the matrix coefficients of the $U^{\varphi(x)}$ form a linear basis of $\mathcal{A}_{2}$.

- $\left\{U^{\varphi(x)} \mid x \in \mathbb{G}\right\}$ is a complete set of irreducible corepresentations of $\left(A_{2}, \Delta_{2}\right)$ and $\varphi$ is a monoidal equivalence of compact quantum groups.

The following result is then a corollary of Theorem 3.9.

Proposition 3.13. Consider two compact quantum groups $\mathbb{G}=(A, \Delta)$ and $\mathbb{G}_{2}=\left(A_{2}, \Delta_{2}\right)$. Let $\varphi: \mathbb{G} \rightarrow \mathbb{G}_{2}$ be a monoidal equivalence. In particular, $\varphi$ is a unitary fiber functor on $\mathbb{G}$.

Denote by $B_{r}$, resp. $B_{u}$ the $C^{*}$-algebras associated to $\varphi$ as in Theorem 3.9, with dense*-subalgebra $\mathcal{B}$. Denote by $\delta$ the corresponding coaction of $(\mathcal{A}, \Delta)$ on $\mathcal{B}$. Denote by $X^{x} \in \mathrm{B}\left(H_{x}, H_{\varphi(x)}\right) \otimes \mathcal{B}$ the unitaries generating $\mathcal{B}$.

- There is a unique coaction $\delta_{2}: \mathcal{B} \rightarrow \mathcal{A}_{2} \otimes \mathcal{B}$ satisfying $\left(\iota \otimes \delta_{2}\right)\left(X^{x}\right)=U_{13}^{\varphi(x)} X_{23}^{x}$ for all $x \in \widehat{\mathbb{G}}$. The coaction $\delta_{2}$ commutes with $\delta$ and extends to $B_{r}$, resp. $B_{u}$, yielding a reduced, resp. universal, ergodic coaction of full quantum multiplicity.

- Every pair of commuting reduced, resp. universal, ergodic coactions of full quantum multiplicity arises in this way from a monoidal equivalence.

Proof. Given the monoidal equivalence $\varphi$, it is obvious to construct the coaction $\delta_{2}$.

It remains to show the second statement. Let $\delta: B \rightarrow B \otimes A$ and $\delta_{2}: B \rightarrow A_{2} \otimes B$ be commuting ergodic coactions of full quantum multiplicity. Denote by $\mathcal{B}$ the unital ${ }^{*}$-subalgebra of $B$ generated by the spectral subspaces of $\delta$. Using Theorem 3.9, we get a unitary fiber functor $\varphi$ on $\mathbb{G}$ and we may assume 
that $\mathcal{B}$ and $\delta$ are constructed from $\varphi$ as in Theorem 3.9. In particular, $\mathcal{B}$ is generated by the coefficients of $X^{x} \in \mathrm{B}\left(H_{x}, H_{\varphi(x)}\right) \otimes \mathcal{B}$.

Because $\delta$ and $\delta_{2}$ commute, the element $\left(\iota \otimes \delta_{2}\right)\left(X^{x}\right)\left(X^{x}\right)_{13}^{*}$ is invariant under $(\iota \otimes \iota \otimes \delta)$. Since $\delta$ is ergodic and $X^{x}$ unitary, we get a unitary element $U^{\varphi(x)} \in B\left(H_{\varphi(x)}\right) \otimes A_{2}$ such that $\left(\iota \otimes \delta_{2}\right)\left(X^{x}\right)=U_{12}^{\varphi(x)} X_{13}^{x}$. Because $\delta_{2}$ is a coaction, we easily compute that $U^{\varphi(x)}$ is a unitary corepresentation of $\mathbb{G}_{2}$.

It remains to show that $\left\{U^{\varphi(x)} \mid x \in \widehat{\mathbb{G}}\right\}$ is a complete set of irreducible unitary corepresentations of $\mathbb{G}_{2}$ and that $\varphi$ is a monoidal equivalence.

Assume that $S \in \operatorname{Mor}(\varphi(x), \varphi(y))$. The element $\left(X^{y}\right)^{*}(S \otimes 1) X^{x} \in \mathrm{B}\left(H_{x}, H_{y}\right) \otimes B$ is invariant under $\iota \otimes \delta_{2}$, so it has the form $T \otimes 1$, with $T \in \mathrm{B}\left(H_{x}, H_{y}\right)$. It follows that $T \in \operatorname{Mor}(x, y)=\delta_{x, y} \mathbb{C}$ and hence, $S \in \delta_{x, y} \mathbb{C}$. So, the $U^{\varphi(x)}$ are mutually inequivalent irreducible corepresentations of $\mathbb{G}_{2}$.

In order to show that the set $\left\{U^{\varphi(x)} \mid x \in \widehat{\mathbb{G}}\right\}$ exhausts all irreducible corepresentations of $\mathbb{G}_{2}$, it suffices to show, for all $a \in \mathcal{A}_{2}$, that $\left(\iota \otimes h_{2}\right)\left((1 \otimes a) U^{\varphi(x)}\right)=0$ for all $x \in \widehat{\mathbb{G}}$, implies $a=0$. But, given the formula for $\delta_{2}$, we get $\left(h_{2} \otimes \iota\right)\left((a \otimes 1) \delta_{2}(x)\right)=0$ for all $x \in B$. Since $\delta_{2}$ is of full quantum multiplicity, this implies that $a=0$.

It remains to show that $\varphi$ is a monoidal equivalence. For this, it suffices to show that $\operatorname{Mor}(\varphi(x), \varphi(y)(T \varphi(z))=$ $\varphi(\operatorname{Mor}(x, y \Subset z))$. If $S \in \operatorname{Mor}\left(x, y(z)\right.$, we use the multiplicativity of $\delta_{2}$ to obtain

$$
\begin{aligned}
(\varphi(S) \otimes 1 \otimes 1) U_{12}^{\varphi(x)} X_{13}^{x} & =\left(\iota \otimes \iota \otimes \delta_{2}\right)\left((\varphi(S) \otimes 1) X^{x}\right)=\left(\iota \otimes \iota \otimes \delta_{2}\right)\left(X_{13}^{y} X_{23}^{z}(S \otimes 1)\right) \\
& =U_{13}^{\varphi(y)} X_{14}^{y} U_{23}^{\varphi(z)} X_{24}^{z}(S \otimes 1 \otimes 1)=U_{13}^{\varphi(y)} U_{23}^{\varphi(z)}(\varphi(S) \otimes 1 \otimes 1) X_{13}^{x} .
\end{aligned}
$$

It follows that $S \in \operatorname{Mor}(\varphi(x), \varphi(y) \oplus \varphi(z))$. The converse inclusion is shown analogously.

\section{Unitary fiber functors preserving the dimension}

We study in this section unitary fiber functors $\varphi$ on a compact quantum group $\mathbb{G}$ preserving the dimension, i.e. satisfying $\operatorname{dim} H_{\varphi(x)}=\operatorname{dim} H_{x}$ for all $x \in \widehat{\mathbb{G}}$. Taking into account Theorem 3.9, this comes down to the study of ergodic coactions of full quantum multiplicity satisfying $\operatorname{mult}(x)=\operatorname{dim}(x)$ for all $x \in \widehat{\mathbb{G}}$.

We establish a relation between unitary fiber functors preserving the dimension (up to isomorphism) and the 2-cohomology of the dual, discrete quantum group $(\hat{A}, \hat{\Delta})$. The following definition is due to Landstad [19] and Wassermann [28], who consider it for the dual of a compact group.

Definition 4.1. A unitary element $\Omega \in \mathrm{M}(\hat{A} \otimes \hat{A})$ is said to be a 2 -cocycle if it satisfies

$$
(\hat{\Delta} \otimes \iota)(\Omega)(\Omega \otimes 1)=(\iota \otimes \hat{\Delta})(\Omega)(1 \otimes \Omega) .
$$

Two 2-cocycles $\Omega_{1}$ and $\Omega_{2}$ are said to differ by a coboundary if there exists a unitary $u \in \mathrm{M}(\hat{A})$ such that $\Omega_{2}=\hat{\Delta}(u) \Omega\left(u^{*} \otimes u^{*}\right)$. We denote this relation by $\Omega_{1} \sim \Omega_{2}$ and observe that $\sim$ is an equivalence relation on the set of 2-cocycles.

Remark 4.2. In the quantum setting, there is no reason that the product of two 2-cocycles is again a 2 -cocycle. So, although we could define the 2-cohomology of $(\hat{A}, \hat{\Delta})$ as the set of equivalence classes of 2 -cocycles, this set has no natural group structure.

Notation 4.3. We denote by $p_{x}, x \in \widehat{\mathbb{G}}$, the minimal central projections of $\hat{A}=\oplus_{x} \mathrm{~B}\left(H_{x}\right)$.

Remark 4.4. Up to coboundary, we can and will assume that a unitary 2-cocycle is normalized, i.e.

$$
\left(p_{\epsilon} \otimes 1\right) \Omega=p_{\epsilon} \otimes 1 \quad \text { and } \quad\left(1 \otimes p_{\epsilon}\right) \Omega=1 \otimes p_{\epsilon} .
$$

Let $\Omega$ be a normalized unitary 2-cocycle on $(\hat{A}, \hat{\Delta})$, the dual of $\mathbb{G}=(A, \Delta)$. Denote

$$
\Omega_{(2)}:=(\hat{\Delta} \otimes \iota)(\Omega)(\Omega \otimes 1)=(\iota \otimes \hat{\Delta})(\Omega)(1 \otimes \Omega) .
$$


It follows from Remark 3.2 that there is a unique unitary fiber functor $\varphi_{\Omega}$ on $\mathbb{G}$ satisfying

$$
H_{\varphi_{\Omega}(x)}=H_{x}, \quad \varphi_{\Omega}(S)=\Omega^{*} S, \quad \varphi_{\Omega}(T)=\Omega_{(2)}^{*} T,
$$

for all $S \in \operatorname{Mor}(x, y \Subset z)$ and $T \in \operatorname{Mor}\left(a, x \uparrow y(z)\right.$. Observe that we implicitly used that $\mathrm{B}\left(H_{y} \otimes H_{z}\right)$ is an ideal in $\mathrm{M}(\hat{A} \otimes \hat{A})$ and hence, $\Omega^{*} S$ is a well defined element of $\mathrm{B}\left(H_{x}, H_{y} \otimes H_{z}\right)$ whenever $S \in \operatorname{Mor}(x, y(z)$.

From Proposition 3.12, we get a compact quantum group $\left(A_{\Omega}, \Delta_{\Omega}\right)$, whose dual $\left(\hat{A}_{\Omega}, \hat{\Delta}_{\Omega}\right)$ is given by

$$
\hat{A}_{\Omega}=\oplus_{x} \mathrm{~B}\left(H_{x}\right)=\hat{A} \quad \text { and } \quad \hat{\Delta}_{\Omega}(a) \varphi_{\Omega}(S)=\varphi_{\Omega}(S) a \quad \text { for all } \quad a \in \mathrm{B}\left(H_{x}\right), S \in \operatorname{Mor}(x, y \circledast z) .
$$

Also, $\varphi_{\Omega}$ becomes a monoidal equivalence between $(A, \Delta)$ and $\left(A_{\Omega}, \Delta_{\Omega}\right)$. Observe that

$$
\hat{\Delta}_{\Omega}(a)=\Omega^{*} \hat{\Delta}(a) \Omega \text { for all } a \in \hat{A}_{\Omega}=\hat{A} .
$$

Proposition 4.5. Let $\varphi$ be a unitary fiber functor on a compact quantum group $\mathbb{G}=(A, \Delta)$ such that $\operatorname{dim} H_{\varphi(x)}=\operatorname{dim} H_{x}$ for all $x \in \widehat{\mathbb{G}}$. Then there exists a normalized unitary 2-cocycle $\Omega$ on $(\hat{A}, \hat{\Delta})$, uniquely determined up to coboundary, such that $\varphi$ is isomorphic with $\varphi_{\Omega}$.

Proof. Denote by $\delta: B \rightarrow B \otimes A$ the reduced ergodic coaction associated with $\varphi$ by Theorem 3.9. Consider the generating unitaries $X^{x} \in \mathrm{B}\left(H_{x}, H_{\varphi(x)}\right) \otimes B$ satisfying $(\iota \otimes \delta)\left(X^{x}\right)=X_{12}^{x} U_{13}^{x}$ for all $x \in \widehat{\mathbb{G}}$.

Since $\operatorname{dim} H_{\varphi(x)}=\operatorname{dim} H_{x}$, we can take unitary elements $u_{x}: H_{\varphi(x)} \rightarrow H_{x}$. Take $u_{\epsilon}=1$. Define $Y^{x}=$ $\left(u_{x} \otimes 1\right) X^{x}$ and consider $Y:=\oplus_{x} Y^{x} \in \mathrm{M}(\hat{A} \otimes B)$. Because the element $(\hat{\Delta} \otimes \iota)(Y) Y_{23}^{*} Y_{13}^{*}$ is invariant under $(\iota \otimes \iota \otimes \delta)$, we find a unitary element $\Omega \in \mathrm{M}(\hat{A} \otimes \hat{A})$ such that

$$
(\hat{\Delta} \otimes \iota)(Y)=(\Omega \otimes 1) Y_{13} Y_{23} .
$$

Applying $\hat{\Delta} \otimes \iota \otimes \iota$ and $\iota \otimes \hat{\Delta} \otimes \iota$ to this equality, we obtain that $\Omega$ is a unitary 2-cocycle on $(\hat{A}, \hat{\Delta})$.

It remains to show that $\varphi$ and $\varphi_{\Omega}$ are isomorphic. Let $S \in \operatorname{Mor}(x, y \uparrow z)$. Then,

$$
\begin{aligned}
(S \otimes 1) Y^{x} & =(\hat{\Delta} \otimes \iota)(Y)(S \otimes 1)=(\Omega \otimes 1) Y_{13} Y_{23}(S \otimes 1)=\left(\Omega\left(u_{y} \otimes u_{z}\right) \otimes 1\right) X_{13}^{y} X_{23}^{z}(S \otimes 1) \\
& =\left(\Omega\left(u_{y} \otimes u_{z}\right) \otimes 1\right)(\varphi(S) \otimes 1) X^{x}=\left(\Omega\left(u_{y} \otimes u_{z}\right) \varphi(S) u_{x}^{*} \otimes 1\right) Y^{x} .
\end{aligned}
$$

Hence, $\varphi_{\Omega}(S)=\Omega^{*} S=\left(u_{y} \otimes u_{z}\right) \varphi(S) u_{x}^{*}$ for all $S \in \operatorname{Mor}(x, y(\tau)$.

It is obvious that $\varphi_{\Omega_{1}}$ is isomorphic with $\varphi_{\Omega_{2}}$ if and only if the 2-cocycles $\Omega_{1}$ and $\Omega_{2}$ differ by a coboundary.

Fix a normalized unitary 2-cocycle $\Omega$ on $(\hat{A}, \hat{\Delta})$ and consider the unitary fiber functor $\varphi_{\Omega}$. Theorem 3.9 yields $\mathrm{C}^{*}$-algebras $B_{r}^{\Omega}$ and $B_{u}^{\Omega}$ with ergodic coactions $\delta_{r}$ and $\delta_{u}$ of full quantum multiplicity. It is, of course, possible to describe these $\mathrm{C}^{*}$-algebras directly in terms of $\Omega$ : they correspond to the $\Omega$-twisted group $\mathrm{C}^{*}$ algebras of $(\hat{A}, \hat{\Delta})$. Before we can prove such a statement, we have to introduce a few notations and a bit of terminology. Such $\Omega$-twisted group $C^{*}$-algebras have been studied by Landstad [19] and Wassermann [28] when $\Omega$ is a unitary 2-cocycle on the dual of a compact group.

Notation 4.6. Denote by $H$ the $L^{2}$-space of the Haar state $h$ on $(A, \Delta)$. We consider $A$ and $\hat{A}$ as being represented on $H$. Denote by $V$ the right regular corepresentation of $(A, \Delta)$. The unitary $V$ is multiplicative in the sense of [1] and belongs to $\mathrm{M}(\hat{A} \otimes A)$. There exists a canonical unitary $u \in \mathrm{B}(H)$ with $u^{2}=1$ such that $\hat{V}:=(u \otimes 1) \Sigma V \Sigma(u \otimes 1)$ is a multiplicative unitary. We know that $u A u \subset A^{\prime}, u \hat{A} u \subset \hat{A}^{\prime}$ and

$$
\hat{\Delta}(x)=V^{*}(1 \otimes x) V=\hat{V}(x \otimes 1) \hat{V}^{*} \quad \text { and } \quad(\iota \otimes \Delta)(V)=V_{12} V_{13},(\iota \otimes \hat{\Delta})(\hat{V})=\hat{V}_{12} \hat{V}_{13} .
$$

The unitaries $V$ and $\hat{V}$ satisfy the pentagonal equation

$$
V_{12} V_{13} V_{23}=V_{23} V_{12} \quad \text { and } \quad \hat{V}_{12} \hat{V}_{13} \hat{V}_{23}=\hat{V}_{23} \hat{V}_{12}
$$


Definition 4.7. An $\Omega$-corepresentation of $(\hat{A}, \hat{\Delta})$ on a Hilbert space $K$ is a unitary $X \in \mathrm{M}(\hat{A} \otimes \mathcal{K}(K))$ satisfying

$$
(\hat{\Delta} \otimes \iota)(X)=(\Omega \otimes 1) X_{13} X_{23} .
$$

The following lemma can be checked immediately using the formulas in Notation 4.6 and in particular, the commutation relation $V_{12} \hat{V}_{23}=\hat{V}_{23} V_{12}$.

Lemma 4.8. Denoting $\widetilde{\Omega}:=(1 \otimes u) \Sigma \Omega^{*} \Sigma(1 \otimes u)$, the unitary $\widetilde{\Omega} V \in \mathrm{M}(\hat{A} \otimes \mathcal{K}(H))$ is an $\Omega$-corepresentation. It is called the right regular $\Omega$-corepresentation.

The next lemma is crucial to define the twisted quantum group $\mathrm{C}^{*}$-algebras.

Lemma 4.9. Let $X$ be an $\Omega$-corepresentation of $(\hat{A}, \hat{\Delta})$ on $K$. Then, $\left[(\mu \otimes \iota)(X) \mid \mu \in \hat{A}^{*}\right]$ is a unital $C^{*}$-algebra.

Proof. Write $B:=\left[(\mu \otimes \iota)(X) \mid \mu \in \hat{A}^{*}\right]$. From the defining relation for an $\Omega$-corepresentation, it follows that $B$ is an algebra acting non-degenerately on $K$. Since $(\hat{\Delta} \otimes \iota)(X) X_{23}^{*}=(\Omega \otimes 1) X_{13}$, we have

$$
\begin{aligned}
B & =\left[(\mu \otimes \eta \otimes \iota)\left((\hat{\Delta} \otimes \iota)(X) X_{23}^{*}\right) \mid \mu, \eta \in \hat{A}^{*}\right]=\left[(\mu \otimes \eta \otimes \iota)\left(\hat{V}_{12} X_{13} \hat{V}_{12}^{*} X_{23}^{*}\right) \mid \mu, \eta \in \hat{A}^{*}\right] \\
& =\left[(\mu \otimes \eta \otimes \iota)\left(X_{13} \hat{V}_{12}^{*} X_{23}^{*}\right) \mid \mu, \eta \in \hat{A}^{*}\right]=\left[(\mu \otimes \eta \otimes \iota)\left(X_{13}\left((1 \otimes \mathcal{K}(H)) \hat{V}^{*}(\mathcal{K}(H) \otimes 1)\right)_{12} X_{23}^{*}\right) \mid \mu, \eta \in \hat{A}^{*}\right] \\
& =\left[(\mu \otimes \eta \otimes \iota)\left(X_{13}(\mathcal{K}(H) \otimes \mathcal{K}(H) \otimes 1) X_{23}^{*}\right) \mid \mu, \eta \in \hat{A}^{*}\right] \\
& =\left[(\mu \otimes \iota)(X)(\eta \otimes \iota)(X)^{*} \mid \mu, \eta \in \hat{A}^{*}\right]=\left[B B^{*}\right] .
\end{aligned}
$$

Here we used the regularity of the multiplicative unitary $\hat{V}$. It follows that $B$ is a $\mathrm{C}^{*}$-algebra. Since $\Omega$ is normalized, we have $\left(p_{\epsilon} \otimes 1\right) X=p_{\epsilon} \otimes 1$ and hence, $B$ is unital.

Definition 4.10. We define the unital $\mathrm{C}^{*}$-algebras

$$
C_{r}^{*}(\mathbb{G}, \Omega):=\left[(\mu \otimes \iota)(\widetilde{\Omega} V) \mid \mu \in \hat{A}^{*}\right] \quad \text { and } \quad C_{u}^{*}(\mathbb{G}, \Omega):=\left[(\mu \otimes \iota)(X) \mid \mu \in \hat{A}^{*}\right],
$$

where $X$ denotes a universal $\Omega$-corepresentation.

Remark that an $\Omega$-corepresentation $X$ on $K$ is said to be universal if for any $\Omega$-corepresentation $Y$ on $K_{1}$, there exists an isometry $v: K_{1} \rightarrow K$ such that $X(1 \otimes v)=(1 \otimes v) Y$. It is clear that a universal $\Omega$-corepresentation exists and that the $\mathrm{C}^{*}$-algebra $C_{u}^{*}(\mathbb{G}, \Omega)$ is well defined up to an isomorphism.

Proposition 4.11. Denote by $\mathcal{B}$ the unital ${ }^{*}$-algebra associated by Theorem 3.9 with the unitary fiber functor $\varphi_{\Omega}$. Consider the unitaries $X^{x} \in \mathrm{B}\left(H_{x}\right) \otimes \mathcal{B}$ generating $\mathcal{B}$. Denote by $B_{r}^{\Omega}$ and $B_{u}^{\Omega}$ the associated reduced and universal $C^{*}$-algebra.

For any $\Omega$-corepresentation $X$ of $(\hat{A}, \hat{\Delta})$ on a Hilbert space $K$, we obtain a representation $\pi$ of $\mathcal{B}$ on $K$ given by $(\iota \otimes \pi)\left(X^{x}\right)=\left(p_{x} \otimes 1\right) X$ for all $x \in \widehat{\mathbb{G}}$. Taking $X=\widetilde{\Omega} V$, we get an isomorphism $B_{r}^{\Omega} \cong C_{r}^{*}(\mathbb{G}, \Omega)$. Taking $X$ to be a universal $\Omega$-corepresentation, we get an isomorphism $B_{u}^{\Omega} \cong C_{u}^{*}(\mathbb{G}, \Omega)$.

Proof. It is immediate that the formula $(\iota \otimes \pi)\left(X^{x}\right)=\left(p_{x} \otimes 1\right) X$ defines a one-to-one correspondence between representations of $\mathcal{B}$ and $\Omega$-corepresentations of $(\hat{A}, \hat{\Delta})$. This already shows the isomorphism $B_{u}^{\Omega} \cong C_{u}^{*}(\mathbb{G}, \Omega)$.

Consider $\pi: \mathcal{B} \rightarrow \mathrm{B}(H)$ given by $(\iota \otimes \pi)\left(X^{x}\right)=\left(p_{x} \otimes 1\right) \widetilde{\Omega} V$. Denote by $\omega$ the unique invariant state on $\mathcal{B}$. To prove the isomorphism $B_{r}^{\Omega} \cong C_{r}^{*}(\mathbb{G}, \Omega)$, it suffices to define a faithful state $\omega_{1}$ on $C_{r}^{*}(\mathbb{G}, \Omega)$ such that $\omega_{1} \pi=\omega$. Define $\alpha: C_{r}^{*}(\mathbb{G}, \Omega) \rightarrow \mathrm{M}(\mathcal{K}(H) \otimes A): \alpha(a)=V(a \otimes 1) V^{*}$. One verifies that $(\iota \otimes \alpha)(\widetilde{\Omega} V)=(\widetilde{\Omega} V)_{12} V_{13}$. Hence, $\alpha: C_{r}^{*}(\mathbb{G}, \Omega) \rightarrow C_{r}^{*}(\mathbb{G}, \Omega) \otimes A$ is a coaction satisfying $\alpha \pi=(\pi \otimes \iota) \delta$. It follows that $(\iota \otimes h) \alpha(a) \in \mathbb{C} 1$ for all $a \in C_{r}^{*}(\mathbb{G}, \Omega)$. So, we can define $\omega_{1}$ by the formula $\omega_{1}(a) 1=(\iota \otimes h) \alpha(a)$. Clearly, $\omega_{1} \pi=\omega$. 


\section{$5 \quad$ Monoidal equivalence for $A_{o}(F)$}

Recall the following definition of the compact quantum group $A_{o}(F)$ [25].

Definition 5.1. For all $n \in \mathbb{N}$ and $F \in \operatorname{GL}(n, C)$ with $F \bar{F}=c 1 \in \mathbb{R} 1, A_{0}(F)$ is defined as the universal compact quantum group generated by the coefficients of the corepresentation $U \in M_{n}(\mathbb{C}) \otimes A_{o}(F)$ with relations

$$
U \text { is unitary and } U=(F \otimes 1) \bar{U}\left(F^{-1} \otimes 1\right) .
$$

Then, $\left(A_{o}(F), U\right)$ is a compact (matrix) quantum group.

When the matrix $F$ has dimension 2, we precisely obtain the quantized versions of the classical Lie group $\mathrm{SU}(2)$, as considered by Woronowicz.

Definition 5.2. ([31]) Let $q \in[-1,1] \backslash\{0\}$. We define $\mathrm{SU}_{q}(2)$ to be the universal $\mathrm{C}^{*}$-algebra generated by 2 elements $\alpha, \gamma$ such that $U=\left(\begin{array}{cc}\alpha & -q \gamma^{*} \\ \gamma & \alpha^{*}\end{array}\right)$ is a unitary corepresentation. Then $\left(\mathrm{SU}_{q}(2), U\right)$ is a compact (matrix) quantum group.

Observe that $\mathrm{SU}_{q}(2) \cong A_{o}\left(\begin{array}{rr}0 & 1 \\ -q^{-1} & 0\end{array}\right)$ and that $\operatorname{dim}_{q}(U)=\left|q+\frac{1}{q}\right|$.

Banica [3] has shown that the irreducible corepresentations of $A_{o}(F)$ can be labelled by $\mathbb{N}$, in such a way that the fusion rules are identical to the fusion rules of the compact Lie group $\operatorname{SU}(2)$. In particular, $\operatorname{Mor}(\epsilon, U \Subset U)$ is one-dimensional and generated by

$$
t_{F}:=\sum_{i} e_{i} \otimes F e_{i}
$$

where $\left(e_{i}\right)$ is the standard basis of $\mathbb{C}^{n}$.

Theorem 5.3. Let $F \in \operatorname{GL}(n, \mathbb{C})$ with $F \bar{F}=c 1$ and $c \in \mathbb{R}$. Consider $\mathbb{G}=A_{o}(F)$.

- Take $F_{1} \in \mathrm{GL}\left(n_{1}, \mathbb{C}\right)$ satisfying $F_{1} \bar{F}_{1}=c_{1} 1$ and $\frac{c}{\operatorname{Tr}\left(F^{*} F\right)}=\frac{c_{1}}{\operatorname{Tr}\left(F_{1}^{*} F_{1}\right)}$. There exists a unitary fiber functor $\varphi_{F_{1}}$ on $\mathbb{G}$, uniquely determined up to isomorphism, such that

$$
\varphi\left(\frac{1}{\sqrt{\operatorname{Tr}\left(F^{*} F\right)}} t_{F}\right)=\frac{1}{\sqrt{\operatorname{Tr}\left(F_{1}^{*} F_{1}\right)}} t_{F_{1}} .
$$

- Every unitary fiber functor $\varphi$ on $\mathbb{G}$ is isomorphic with one of the form $\varphi_{F_{1}}$. Moreover, $\varphi_{F_{1}}$ is isomorphic with $\varphi_{F_{2}}$ if and only if $n_{1}=n_{2}$ and there exists a unitary $v \in \mathrm{U}\left(n_{1}\right)$ and a $\lambda \neq 0$ such that $F_{2}=\lambda v F_{1} v^{t}$.

Proof. Take a parameter $\beta \in \mathbb{R} \backslash\{0\}$. Consider the universal graded $\mathrm{C}^{*}$-algebra $(A(n, m))_{n, m \in \mathbb{N}}$ satisfying $A_{n, m}=\{0\}$ if $n-m$ is odd and generated by elements $v(r, s) \in A(r+s, r+s+2)$ with relations (denoting $1_{n}$ the unit of the $\mathrm{C}^{*}$-algebra $\left.A(n):=A(n, n)\right)$

$$
\begin{aligned}
v(r, s)^{*} v(r, s) & =1_{r+s} \\
v(r, s+1)^{*} v(r+1, s) & =\beta 1_{r+s+1} \\
v(r, k+l+2) v(r+k, l) & =v(r+k+2, l) v(r, k+l) \\
v(r, k+l+2)^{*} v(r+k+2, l) & =v(r+k, l) v(r, k+l)^{*}
\end{aligned}
$$

Take $F \in \operatorname{GL}(n, \mathbb{C})$ with $F \bar{F}=c 1$ and $c \in \mathbb{R}$. Put $\beta=\frac{c}{\operatorname{Tr}\left(F^{*} F\right)}$. Let $\mathbb{G}=\left(A_{o}(F), \Delta\right)$ and denote by $U^{n}$ the $n$-fold tensor product of the fundamental corepresentation, with the convention that $U^{0}=\epsilon$. Take the isometric $t \in \operatorname{Mor}\left(\epsilon, U^{2}\right)$ given by $t=\frac{1}{\sqrt{\operatorname{Tr}\left(F^{*} F\right)}} t_{F}$. Then, $\left(t^{*} \otimes 1\right)(1 \otimes t)=\beta 1$.

We get a natural ${ }^{*}$-homomorphism

$$
\pi:(A(n, m))_{n, m \in \mathbb{N}} \rightarrow\left(\operatorname{Mor}\left(U^{n}, U^{m}\right)\right)_{n, m \in \mathbb{N}}
$$


given by $\pi(v(r, s))=1_{r} \otimes t \otimes 1_{s}$. Because of Proposition 1 in [2], $\pi$ is surjective. It follows from the comments after Théorème 4 in [3], that $\pi$ is faithful on $A(n, n)$ for all $n$. But then, $\pi$ is faithful on $A(n, m)$ because

$$
\pi(T)=0 \Leftrightarrow \pi\left(T^{*} T\right)=0 \Leftrightarrow T^{*} T=0 \Leftrightarrow T=0 .
$$

We conclude that $\pi$ is a ${ }^{*}$-isomorphism.

Take $F_{1} \in \operatorname{GL}\left(n_{1}, \mathbb{C}\right)$ satisfying $F_{1} \bar{F}_{1}=c_{1} 1$ and $\frac{c}{\operatorname{Tr}\left(F^{*} F\right)}=\frac{c_{1}}{\operatorname{Tr}\left(F_{1}^{*} F_{1}\right)}$. Write $K=\mathbb{C}^{n_{1}}$ and denote by $K^{n}$ the $n$-fold tensor product of $K$, with the convention that $K^{0}=\mathbb{C}$. From the preceding discussion, we obtain a faithful *-homomorphism

$$
\pi:\left(\operatorname{Mor}\left(U^{n}, U^{m}\right)\right)_{n, m \in \mathbb{N}} \rightarrow\left(\mathrm{B}\left(K^{n}, K^{m}\right)\right)_{n, m \in \mathbb{N}}
$$

satisfying $\pi(t)=\frac{1}{\sqrt{\operatorname{Tr}\left(F_{1}^{*} F_{1}\right)}} t_{F_{1}}, \pi(1)=1$ and $\pi(1 \otimes T \otimes 1)=1 \otimes \pi(T) \otimes 1$ for all $T$.

We choose a concrete identification $\widehat{\mathbb{G}}=\mathbb{N}$ as follows. We define $P_{n} \in \operatorname{Mor}\left(U^{n}, U^{n}\right)$ as the unique projection satisfying $P_{n} T=0$ for all $r<n$ and all $T \in \operatorname{Mor}\left(U^{r}, U^{n}\right)$. We define $U_{n}$ as the restriction of $U^{n}$ to the image of $P_{n}$. We then identify

$$
\operatorname{Mor}\left(n_{1} \uparrow \cdots \oplus n_{r}, m_{1} \top \cdots \oplus m_{k}\right)=\left(P_{m_{1}} \otimes \cdots \otimes P_{m_{k}}\right) \operatorname{Mor}\left(U^{n_{1}+\cdots+n_{r}}, U^{m_{1}+\cdots+m_{k}}\right)\left(P_{n_{1}} \otimes \cdots \otimes P_{n_{r}}\right) .
$$

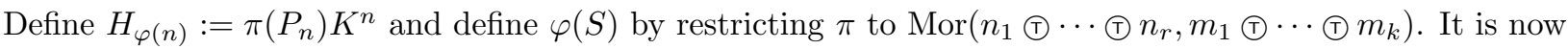
obvious that $\varphi$ is a unitary fiber functor on $\mathbb{G}$.

Suppose conversely that $\varphi$ is a unitary fiber functor on $A_{o}(F)$. We continue to use the concrete identification $\widehat{\mathbb{G}}=\mathbb{N}$ introduced above. Up to isomorphism, we may assume that $H_{\varphi(1)}=\mathbb{C}^{n_{1}}$ and we denote $K=\mathbb{C}^{n_{1}}$. We define the ${ }^{*}$-homomorphism $\pi:\left(\operatorname{Mor}\left(U^{n}, U^{m}\right)\right)_{n, m} \rightarrow\left(\mathrm{B}\left(K^{n}, K^{m}\right)\right)_{n, m}$ by restricting $\varphi$. Define the matrix $F_{1}$ such that $\varphi\left(t_{F}\right)=t_{F_{1}}$. Then, $F_{1} \bar{F}_{1}=c_{1} 1$ with $c_{1}=c$ and $\operatorname{Tr}\left(F_{1}^{*} F_{1}\right)=\operatorname{Tr}\left(F^{*} F\right)$. Since $t$ generates the graded $\mathrm{C}^{*}$-algebra $\left(\operatorname{Mor}\left(U^{n}, U^{m}\right)\right)_{n, m}, \pi$ coincides with the ${ }^{*}$-homomorphism constructed in the first part of the proof starting with $F_{1}$. Denoting by $T_{n} \in \operatorname{Mor}(n, 1 \oplus \cdots \oplus 1)$ the embedding, we get unitary operators $\varphi\left(T_{n}\right): H_{\varphi(n)} \rightarrow \pi\left(P_{n}\right) K^{n}$ who implement the isomorphism between $\varphi$ and $\varphi_{F_{1}}$.

Corollary 5.4. Let $F \in \mathrm{GL}(n, \mathbb{C})$ with $F \bar{F}=c 1$ and $c \in \mathbb{R}$. Consider $\mathbb{G}=A_{o}(F)$. A compact quantum group $\mathbb{G}_{1}$ is monoidally equivalent with $\mathbb{G}$ if and only if there exists $F_{1} \in \mathrm{GL}\left(n_{1}, \mathbb{C}\right)$ satisfying $F_{1} \bar{F}_{1}=c_{1} 1$ and $\frac{c}{\operatorname{Tr}\left(F^{*} F\right)}=\frac{c_{1}}{\operatorname{Tr}\left(F_{1}^{*} F_{1}\right)}$ such that $\mathbb{G}_{1} \cong A_{o}\left(F_{1}\right)$.

Proof. The unitary fiber functor $\varphi_{F_{1}}$ constructed in Theorem 5.3 yields a monoidal equivalence $A_{o}(F) \underset{\text { mon }}{\sim}$ $A_{o}\left(F_{1}\right)$. Since these fiber functors $\varphi_{F_{1}}$ are, up to isomorphism, the only unitary fiber functors on $A_{o}(F)$, we are done.

So, we exactly know when the compact quantum groups $A_{o}\left(F_{1}\right)$ and $A_{o}\left(F_{2}\right)$ are monoidally equivalent. If this is the case, Proposition 3.13 provides us with a universal $\mathrm{C}^{*}$-algebra $B_{u}$ and a pair of ergodic coactions of full quantum multiplicity. It is possible to give an intrinsic description of this $\mathrm{C}^{*}$-algebra $B_{u}$.

Theorem 5.5. Let $F_{i} \in \mathrm{GL}\left(n_{i}, \mathbb{C}\right)$ by such that $F_{i} \bar{F}_{i}=c_{i} 1$ for $c_{i} \in \mathbb{R}(i=1,2)$. Assume that $c_{1}=c_{2}$ and $\operatorname{Tr}\left(F_{1}^{*} F_{1}\right)=\operatorname{Tr}\left(F_{2}^{*} F_{2}\right)$.

- Denote by $A_{o}\left(F_{1}, F_{2}\right)$ the universal unital $C^{*}$-algebra generated by the coefficients of

$$
Y \in M_{n_{2}, n_{1}}(\mathbb{C}) \otimes A_{o}\left(F_{1}, F_{2}\right) \quad \text { with relations } \quad Y \quad \text { unitary } \quad \text { and } \quad Y=\left(F_{2} \otimes 1\right) \bar{Y}\left(F_{1}^{-1} \otimes 1\right) .
$$

Then, $A_{o}\left(F_{1}, F_{2}\right) \neq 0$ and there exists a unique pair of commuting universal ergodic coactions of full quantum multiplicity, $\delta_{1}$ of $A_{o}\left(F_{1}\right)$ and $\delta_{2}$ of $A_{o}\left(F_{2}\right)$, such that

$$
\left(\iota \otimes \delta_{1}\right)(Y)=Y_{12}\left(U_{1}\right)_{13} \quad \text { and } \quad\left(\iota \otimes \delta_{2}\right)(Y)=\left(U_{2}\right)_{12} Y_{13} .
$$

Here, $U_{i}$ denotes the fundamental corepresentation of $A_{o}\left(F_{i}\right)$. 
- $\left(A_{o}\left(F_{1}, F_{2}\right), \delta_{1}, \delta_{2}\right)$ is isomorphic with the $C^{*}$-algebra $B_{u}$ and the coactions thereon given by Proposition 3.13 and the monoidal equivalence $A_{o}\left(F_{1}\right) \underset{\text { mon }}{\sim} A_{o}\left(F_{2}\right)$ of Corollary 5.4.

- The multiplicity of the fundamental corepresentation $U_{1}$ in the coaction $\delta_{1}$ equals $n_{2}$.

Remark that the condition on the matrices $F_{1}$ and $F_{2}$ is not really less general then the condition in Theorem 5.3, but just a normalization: if $\frac{c_{1}}{\operatorname{Tr}\left(F_{1}^{*} F_{1}\right)}=\frac{c_{2}}{\operatorname{Tr}\left(F_{2}^{*} F_{2}\right)}$, we multiply $F_{2}$ by a scalar and obtain $c_{1}=c_{2}$ and $\operatorname{Tr}\left(F_{1}^{*} F_{1}\right)=\operatorname{Tr}\left(F_{2}^{*} F_{2}\right)$.

Proof. Take $F_{i}$ as in the statement of the theorem and denote by $\varphi$ the unitary fiber functor on $\mathbb{G}_{1}:=A_{o}\left(F_{1}\right)$ given by Theorem 5.3 and (5.1). We continue to use the identification of $\widehat{\mathbb{G}}_{1}$ with $\mathbb{N}$. Theorem 3.9 provides us with a ${ }^{*}$-algebra $\mathcal{B}$ generated by the coefficients of unitary operators $X^{n} \in \mathrm{B}\left(H_{n}, H_{\varphi(n)}\right) \otimes \mathcal{B}, n \in \mathbb{N}$. Define $X:=X^{1}$. Since every element of $\widehat{\mathbb{G}}_{1}$ appears in a tensor power of the fundamental corepresentation and since $\overline{1}=1$, it follows that the coefficients of $X$ generate $\mathcal{B}$ as an algebra. Moreover (3.7) precisely says that $X=\left(F_{2} \otimes 1\right) \bar{X}\left(F_{1}^{-1} \otimes 1\right)$. It follows that $A_{o}\left(F_{1}, F_{2}\right) \neq 0$. Denoting by $\mathcal{C}$ the unital ${ }^{*}$-subalgebra of $A_{o}\left(F_{1}, F_{2}\right)$ generated by the coefficients of $Y$, we get a surjective ${ }^{*}$-homomorphism $\rho: \mathcal{C} \rightarrow \mathcal{B}$ satisfying $(\iota \otimes \rho)(Y)=X$. It remains to show that $\rho$ is a ${ }^{*}$-isomorphism.

Denote by $U$ the fundamental corepresentation of $A_{o}\left(F_{1}\right)$ on $H=\mathbb{C}^{n_{1}}$ and by $U^{n}$ its $n$-th tensor power, on $H^{n}$. As in the proof of Theorem 5.3, we denote by $P_{n} \in \operatorname{Mor}\left(U^{n}, U^{n}\right)$ the projection onto the irreducible corepresentation $U_{n}$. Denote $K=\mathbb{C}^{n_{2}}$ and denote by $K^{n}$ the $n$-th tensor power of $K$. Recall that we constructed a faithful *-homomorphism $\pi:\left(\operatorname{Mor}\left(U^{n}, U^{m}\right)\right)_{n, m} \rightarrow\left(\mathrm{B}\left(K^{n}, K^{m}\right)\right)_{n, m}$ and that $\varphi$ is defined by restricting $\pi$ to the relevant subspaces. The graded $\mathrm{C}^{*}$-algebra $\left(\operatorname{Mor}\left(U^{n}, U^{m}\right)\right)_{n, m}$ is generated by the elements $t \in \operatorname{Mor}\left(\epsilon, U^{2}\right)$ and $1_{r} \otimes t \otimes 1_{s}$.

Put $Y^{n}=Y_{1, n+1} \cdots Y_{n, n+1} \in \mathrm{B}\left(H^{n}, K^{n}\right) \otimes A_{o}\left(F_{1}, F_{2}\right)$. Since $Y^{2}(t \otimes 1)=\pi(t) \otimes 1$, it follows that $Y^{m}(S \otimes 1)=$ $(\pi(S) \otimes 1) Y^{n}$ for all $S \in \operatorname{Mor}\left(U^{n}, U^{m}\right)$. Define a linear map $\gamma: \mathcal{B} \rightarrow \mathcal{C}$ by the formula $(\iota \otimes \gamma)\left(X^{n}\right)=$ $Y^{n}\left(P_{n} \otimes 1\right)=\left(\pi\left(P_{n}\right) \otimes 1\right) Y^{n}$. We claim that $\gamma$ is multiplicative. Take $a, n, m \in \mathbb{N}$ and $S \in \operatorname{Mor}(a, n \uparrow m)$. Then,

$$
\begin{aligned}
(\iota \otimes \iota \otimes \gamma)\left(X_{13}^{n} X_{23}^{m}\right)(S \otimes 1) & =(\varphi(S) \otimes 1)(\iota \otimes \gamma)\left(X^{a}\right) \\
& =(\varphi(S) \otimes 1)\left(P_{a} \otimes 1\right) Y^{a} \\
& =(\pi(S) \otimes 1) Y^{a}=Y^{n+m}(S \otimes 1) \\
& =\left(Y^{n}\left(P_{n} \otimes 1\right)\right)_{13}\left(Y^{m}\left(P_{m} \otimes 1\right)\right)_{23}(S \otimes 1) \\
& =(\iota \otimes \gamma)\left(X^{n}\right)_{13}(\iota \otimes \gamma)\left(X^{m}\right)_{23}(S \otimes 1) .
\end{aligned}
$$

Because $(\iota \otimes \gamma \rho)(Y)=Y$, because $\gamma$ is multiplicative and because the coefficients of $Y$ generate $\mathcal{C}$ as an algebra, it follows that $\gamma$ is the inverse of $\rho$. So, $\rho$ is indeed a ${ }^{*}$-isomorphism.

Remark 5.6. A combination of Proposition 6.2.6 in [6] and the results in [7] yields an alternative proof for the fact that $A_{o}\left(F_{1}, F_{2}\right) \neq 0$.

Remark 5.7. Combining the co-amenability of $\mathrm{SU}_{q}(2)$ with Remark 2.6, we obtain the following. If either $F_{1}$ or $F_{2}$ is a two by two matrix (but not necessarily both), the (unique) invariant state on the universal $\mathrm{C}^{*}$-algebra $A_{o}\left(F_{1}, F_{2}\right)$ is faithful. So, the ergodic coactions of $A_{o}\left(F_{1}\right)$ and $A_{o}\left(F_{2}\right)$ are both universal and reduced. This is somehow remarkable, because $A_{o}(F)$ is not co-amenable when $F$ has dimension strictly bigger than 2 .

Moreover, still supposing that either $F_{1}$ or $F_{2}$ is a two by two matrix, we also get that $A_{o}\left(F_{1}, F_{2}\right)$ is a nuclear $\mathrm{C}^{*}$-algebra. Indeed, supposing that $F_{2} \in \mathrm{GL}(2, \mathbb{C}), A_{o}\left(F_{1}, F_{2}\right)$ is Morita equivalent with its double crossed product, i.e. the crossed product of the compact operators with the co-amenable quantum group $A_{o}\left(F_{2}\right)$, yielding a nuclear $\mathrm{C}^{*}$-algebra. See [13] for details.

A precise parameterisation of the unitary fiber functors (and hence, the ergodic coactions of full quantum multiplicity) on the quantum groups $A_{o}(F)$, amounts to the study of matrices $F \in \operatorname{GL}(n, \mathbb{C})$ satisfying $F \bar{F}= \pm 1$, up to the equivalence relation

$$
F_{1} \sim F \quad \text { if and only if there exists a unitary } v \in \mathrm{U}(n) \text { such that } F_{1}=v F v^{t} .
$$


Let $F \in \operatorname{GL}(n, \mathbb{C})$ with $F \bar{F}= \pm 1$. Denote $H=\mathbb{C}^{n}$ and $J: H \rightarrow H$ the complex conjugation. We rather look at the anti-linear operator $\mathcal{F}=J F$, satisfying $\mathcal{F}^{2}= \pm 1$. In the case where $\mathcal{F}^{2}=1$, our data come down to giving a real vector space together with a Hilbert space structure on the complexification. In the case where $\mathcal{F}^{2}=-1, H$ becomes a right module on the quaternions such that, restricting the quaternions to $\mathbb{C}$, we get a Hilbert space. In particular $H$ is even-dimensional.

It is then straightforward to provide a fundamental domain for the equivalence relation (5.2) (see e.g. [33]). Take $F \in \operatorname{GL}(n, \mathbb{C})$ with $F \bar{F}= \pm 1$. Let $\mathcal{F}=\mathcal{J}|F|$ be the polar decomposition of $\mathcal{F}$. Then, $\mathcal{J}$ is an antiunitary, $\mathcal{J}^{2}= \pm 1$ and $\mathcal{J}|F| \mathcal{J}^{*}=|F|^{-1}$. Define $H_{<}$as the subspace of $H$ spanned by the eigenvectors of $|F|$ with eigenvalue $\lambda<1$. Define $H_{>}=\mathcal{J} H_{<}$, which is as well the subspace of $H$ spanned by the eigenvectors of $|F|$ with eigenvalue $\lambda>1$. Finally, let $H_{1}$ be the subset of eigenvectors of $|F|$ with eigenvalue 1 . Take an orthonormal basis $\xi_{1}, \ldots, \xi_{k}$ of $H_{<}$of eigenvectors of $|F|$ with eigenvalues $0<\lambda_{1} \leq \cdots \leq \lambda_{k}<1$.

If $F \bar{F}=1$, we have $\mathcal{J}^{2}=1$ and we take an orthonormal basis $\mu_{1}, \ldots, \mu_{n-2 k}$ for $H_{1}$ of real vectors: $\mathcal{J} \mu_{i}=\mu_{i}$. If $\left(e_{i}\right)$ denotes the standard basis for $\mathbb{C}^{n}$ and $w:\left(e_{i}\right) \rightarrow\left(\xi_{i}, \mathcal{J} \xi_{i}, \mu_{i}\right)$ denotes the transition unitary, we find that

$$
w^{t} F w=\left(\begin{array}{ccc}
0 & D\left(\lambda_{1}, \ldots, \lambda_{k}\right) & 0 \\
D\left(\lambda_{1}, \ldots, \lambda_{k}\right)^{-1} & 0 & 0 \\
0 & 0 & 1_{n-2 k}
\end{array}\right) .
$$

Here, $D\left(\lambda_{1}, \ldots, \lambda_{k}\right)$ denotes the diagonal matrix with the $\lambda_{i}$ along the diagonal.

If $F \bar{F}=-1$, we have $\mathcal{J}^{2}=-1, H_{1}$ has even dimension and we take an orthonormal basis $\mu_{1}, \ldots, \mu_{r}$, $\mathcal{J} \mu_{1}, \ldots, \mathcal{J} \mu_{r}$ for $H_{1}$. If $w:\left(e_{i}\right) \rightarrow\left(\xi_{i}, \mu_{i}, \mathcal{J} \xi_{i}, \mathcal{J} \mu_{i}\right)$ denotes the transition unitary, we find that

$$
w^{t} F w=\left(\begin{array}{cc}
0 & D\left(\lambda_{1}, \ldots, \lambda_{n / 2}\right) \\
-D\left(\lambda_{1}, \ldots, \lambda_{n / 2}\right)^{-1} & 0
\end{array}\right),
$$

where $0<\lambda_{1} \leq \cdots \leq \lambda_{n / 2} \leq 1$

Since the spectrum of $F^{*} F$ is invariant under the equivalence relation (5.2), a fundamental domain is given by the matrices in (5.3) with $2 k \leq n$ and $0<\lambda_{1} \leq \cdots \leq \lambda_{k}<1$ and the matrices in (5.4) with $0<\lambda_{1} \leq$ $\cdots \leq \lambda_{n / 2} \leq 1$.

Corollary 5.8. Let $0<q \leq 1$. For every even natural number $n$ with $2 \leq n \leq q+\frac{1}{q}$, the quantum group $\mathrm{SU}_{q}(2)$ admits an ergodic coaction of full quantum multiplicity such that the multiplicity of the fundamental corepresentation is $n$. If $-1 \leq q<0$, the same statement holds for every natural number $n$ with $2 \leq n \leq$ $\left|q+\frac{1}{q}\right|$.

Corollary 5.9. Let $F \in \operatorname{GL}(n, \mathbb{C})$ with $F \bar{F}=c 1$ and $c \in \mathbb{R}$. Denote $\mathbb{G}=A_{o}(F)$. For all $F_{1} \in \mathrm{GL}\left(n_{1}, \mathbb{C}\right)$ satisfying $F_{1} \bar{F}_{1}=c 1$ and $\operatorname{Tr}\left(F_{1}^{*} F_{1}\right)=\operatorname{Tr}\left(F^{*} F\right)$, we denote by $\delta_{F_{1}}$ the coaction of $\mathbb{G}$ on $A_{o}\left(F, F_{1}\right)$ defined in Theorem 5.5.

- Up to isomorphism, the $\delta_{F_{1}}$ yield all universal ergodic coactions of full quantum multiplicity of $\mathbb{G}$. Moreover, $\delta_{F_{1}}$ is isomorphic with $\delta_{F_{2}}$ if and only if $n_{1}=n_{2}$ and there exists a unitary $v \in \mathrm{U}\left(n_{1}\right)$ such that $F_{2}=v F_{1} v^{t}$.

- For all $F_{1}$ as above with $n_{1}=n$, we denote by $\Omega\left(F_{1}\right)$ the unitary 2 -cocycle on the dual of $\mathbb{G}$ associated with the unitary fiber functor $\varphi_{F_{1}}$. The $\Omega\left(F_{1}\right)$ describe, up to coboundary, all unitary 2-cocycles on the dual of $\mathbb{G}$. Moreover $\Omega\left(F_{1}\right)$ and $\Omega\left(F_{2}\right)$ differ by a coboundary if and only if there exists a unitary $v \in \mathrm{U}(n)$ such that $F_{2}=v F_{1} v^{t}$.

Corollary 5.10. Every unitary 2-cocycle on the dual of $\mathrm{SU}_{q}(2)$ is a coboundary.

\section{Monoidal equivalence for $A_{u}(F)$}

In this section, we prove, for the unitary quantum groups $A_{u}(F)$ studied by Banica [3], analogous results as for $A_{o}(F)$. 
Again, we give a complete classification of unitary fiber functors, monoidally equivalent quantum groups, ergodic coactions of full quantum multiplicity and 2-cohomology.

Recall the following definition.

Definition 6.1. For all $n \in \mathbb{N}$ and $F \in \operatorname{GL}(n, \mathbb{C})$, we define $A_{u}(F)$ as the universal compact quantum group generated by the coefficients of the corepresentation $U \in M_{n}(\mathbb{C}) \otimes A_{u}(F)$ with relations

$$
U \text { and }(F \otimes 1) \bar{U}\left(F^{-1} \otimes 1\right) \text { are unitary . }
$$

Then, $\left(A_{u}(F), U\right)$ is a compact (matrix) quantum group.

Banica [3] has shown that the irreducible corepresentations of $A_{u}(F)$ can be labelled by the free monoid $\mathbb{N} \star \mathbb{N}$ generated by $\alpha$ and $\beta$. He also computed the corresponding fusion rules.

Defining $U^{\alpha}:=U$ and $U^{\beta}:=(F \otimes 1) \bar{U}\left(F^{-1} \otimes 1\right)$, the spaces $\operatorname{Mor}\left(\epsilon, U^{\alpha} \odot U^{\beta}\right)$ and $\operatorname{Mor}\left(\epsilon, U^{\beta} \oplus U^{\alpha}\right)$ are one-dimensional and generated by

$$
t_{F}:=\sum_{i} e_{i} \otimes F e_{i} \quad \text { resp. } \quad s_{F}:=\sum_{i} e_{i} \otimes \bar{F}^{-1} e_{i}
$$

Theorem 6.2. Let $F \in \mathrm{GL}(n, \mathbb{C})$ be normalized such that $\operatorname{Tr}\left(F^{*} F\right)=\operatorname{Tr}\left(\left(F^{*} F\right)^{-1}\right)$. Let $\mathbb{G}=A_{u}(F)$.

- If $F_{1} \in \mathrm{GL}\left(n_{1}, \mathbb{C}\right)$ satisfies $\operatorname{Tr}\left(F_{1}^{*} F_{1}\right)=\operatorname{Tr}\left(\left(F_{1}^{*} F_{1}\right)^{-1}\right)=\operatorname{Tr}\left(F^{*} F\right)$, there exists a unitary fiber functor $\varphi_{F_{1}}$ on $\mathbb{G}$, uniquely determined up to isomorphism, such that

$$
\varphi\left(t_{F}\right)=t_{F_{1}} \quad \text { and } \quad \varphi\left(s_{F}\right)=s_{F_{1}} .
$$

- Every unitary fiber functor $\varphi$ on $\mathbb{G}$ is isomorphic with one of the form $\varphi_{F_{1}}$. Moreover, $\varphi_{F_{1}}$ is isomorphic with $\varphi_{F_{2}}$ if and only if $n_{1}=n_{2}$ and there exist unitary elements $v, w \in \mathrm{U}\left(n_{1}\right)$ such that $F_{2}=v F_{1} w$.

Proof. Let $\mathbb{N} \star \mathbb{N}$ be the free monoid generated by $\alpha$ and $\beta$. Denote by $e$ the empty word. Elements of $\mathbb{N} \star \mathbb{N}$ are words in $\alpha$ and $\beta$.

Take a parameter $c>0$. Let $(A(p, q))_{p, q \in \mathbb{N} \star \mathbb{N}}$ be the universal graded $\mathrm{C}^{*}$-algebra generated by elements

$$
V_{x}(p, q) \in A(p q, p x q) \quad \text { for } \quad p, q \in \mathbb{N} \star \mathbb{N}, \quad x \in\{\alpha \beta, \beta \alpha\}
$$

with relations (denoting by $1_{p}$ the unit of the $\mathrm{C}^{*}$-algebra $A(p):=A(p, p)$ )

$$
\begin{aligned}
V_{x}(p, q)^{*} V_{x}(p, q) & =1_{p q} \\
V_{\alpha \beta}(p, \alpha q)^{*} V_{\beta \alpha}(p \alpha, q) & =c 1_{p \alpha q} \\
V_{\beta \alpha}(p, \beta q)^{*} V_{\alpha \beta}(p \beta, q) & =c 1_{p \beta q} \\
V_{y}(p, q x r) V_{x}(p q, r) & =V_{x}(p y q, r) V_{y}(p, q r) \\
V_{x}(p, q y r)^{*} V_{y}(p x q, r) & =V_{y}(p q, r) V_{x}(p, q r)^{*}
\end{aligned}
$$

Take $F \in \operatorname{GL}(n, \mathbb{C})$ normalized in such a way that $\operatorname{Tr}\left(F^{*} F\right)=\operatorname{Tr}\left(\left(F^{*} F\right)^{-1}\right)$. Put $c=\operatorname{Tr}\left(F^{*} F\right)$. Consider the quantum group $\mathbb{G}=\left(A_{u}(F), \Delta\right)$. Define for every $p \in \mathbb{N} \star \mathbb{N}$ the unitary corepresentation $U^{p}$ of $\mathbb{G}$ inductively by $U^{p q}:=U^{p} \odot U^{q}$.

Defining $t \in \operatorname{Mor}\left(\epsilon, U^{\alpha \beta}\right)$ and $s \in \operatorname{Mor}\left(\epsilon, U^{\beta \alpha}\right)$ by the formulas $t=\frac{1}{\sqrt{\operatorname{Tr}\left(F^{*} F\right)}} t_{F}$ and $s=\frac{1}{\sqrt{\operatorname{Tr}\left(F^{*} F\right)}} s_{F}$, we get a natural *-homomorphism

$$
\pi:(A(p, q))_{p, q \in \mathbb{N} \star \mathbb{N}} \rightarrow\left(\operatorname{Mor}\left(U^{p}, U^{q}\right)\right)_{p, q \in \mathbb{N} \star \mathbb{N}}
$$

given by $\pi\left(V_{\alpha \beta}(p, q)\right)=1_{p} \otimes t \otimes 1_{q}$ and $\pi\left(V_{\beta \alpha}(p, q)\right)=1_{p} \otimes s \otimes 1_{q}$. It follows from Proposition 4 , the proof of Théorème 1 and Proposition 3 in [3] that $\pi$ is an isomorphism of $\mathrm{C}^{*}$-algebras. 
Take $F_{1} \in \operatorname{GL}\left(n_{1}, \mathbb{C}\right)$ satisfying $\operatorname{Tr}\left(F_{1}^{*} F_{1}\right)=\operatorname{Tr}\left(\left(F_{1}^{*} F_{1}\right)^{-1}\right)=\operatorname{Tr}\left(F^{*} F\right)$. Write $K^{\alpha}=K^{\beta}=\mathbb{C}^{n_{1}}$ and define inductively $K^{p}$, for all $p \in \mathbb{N} \star \mathbb{N}$ such that $K^{p q}=K^{p} \otimes K^{q}$. We take $K^{e}=\mathbb{C}$. From the preceding discussion, we obtain a faithful *-homomorphism

$$
\pi:\left(\operatorname{Mor}\left(U^{p}, U^{q}\right)\right)_{p, q \in \mathbb{N} \star \mathbb{N}} \rightarrow\left(\mathrm{B}\left(K^{p}, K^{q}\right)\right)_{p, q \in \mathbb{N} \star \mathbb{N}}
$$

satisfying $\pi(t)=\frac{1}{\sqrt{\operatorname{Tr}\left(F_{1}^{*} F_{1}\right)}} t_{F_{1}}, \pi(s)=\frac{1}{\sqrt{\operatorname{Tr}\left(F_{1}^{*} F_{1}\right)}} s_{F_{1}}$ and $\pi(1 \otimes S \otimes 1)=1 \otimes \pi(S) \otimes 1$ for all $S$.

We choose a concrete identification of $\widehat{\mathbb{G}}$ with $\mathbb{N} \star \mathbb{N}$ as follows. We define, for $p \in \mathbb{N} \star \mathbb{N}, P_{p} \in \operatorname{Mor}\left(U^{p}, U^{p}\right)$ as the unique projection satisfying $P_{p} T=0$ for all $r \in \mathbb{N} \star \mathbb{N}$ with length $r<$ length $p$ and all $T \in \operatorname{Mor}\left(U^{r}, U^{p}\right)$. We define $U_{p}$ as the restriction of $U^{p}$ to the image of $P_{p}$. We then identify

$$
\operatorname{Mor}\left(p_{1} \uparrow \cdots \odot p_{r}, q_{1} \uparrow \cdots \odot q_{k}\right)=\left(P_{q_{1}} \otimes \cdots \otimes P_{q_{k}}\right) \operatorname{Mor}\left(U^{p_{1} \cdots p_{r}}, U^{q_{1} \cdots q_{k}}\right)\left(P_{p_{1}} \otimes \cdots \otimes P_{p_{r}}\right) .
$$

Define $H_{\varphi(p)}:=\pi\left(P_{p}\right) K^{p}$ and define $\varphi(S)$ by restricting $\pi$ to $\operatorname{Mor}\left(p_{1} \uparrow \cdots \odot p_{r}, q_{1} \uparrow \cdots \odot q_{k}\right)$. It is obvious that $\varphi$ is a unitary fiber functor.

The converse statement is proven in exactly the same way as in the proof of Theorem 5.3.

The next two results are proven in exactly the same way as the corresponding results for $A_{o}(F)$.

Corollary 6.3. Let $F \in \operatorname{GL}(n, \mathbb{C})$ with $\operatorname{Tr}\left(F^{*} F\right)=\operatorname{Tr}\left(\left(F^{*} F\right)^{-1}\right)$ and consider $\mathbb{G}=A_{o}(F)$. A compact quantum group $\mathbb{G}_{1}$ is monoidally equivalent with $\mathbb{G}$ if and only if there exists $F_{1} \in \mathrm{GL}\left(n_{1}, \mathbb{C}\right)$ satisfying $\operatorname{Tr}\left(F_{1}^{*} F_{1}\right)=\operatorname{Tr}\left(\left(F_{1}^{*} F_{1}\right)^{-1}\right)=\operatorname{Tr}\left(F^{*} F\right)$ such that $\mathbb{G}_{1} \cong A_{o}\left(F_{1}\right)$.

So, we exactly know when the compact quantum groups $A_{u}\left(F_{1}\right)$ and $A_{u}\left(F_{2}\right)$ are monoidally equivalent. If this is the case, Proposition 3.13 provides us with a universal $\mathrm{C}^{*}$-algebra $B_{u}$ and a pair of ergodic coactions of full quantum multiplicity. It is again possible to give an intrinsic description of this $\mathrm{C}^{*}$-algebra $B_{u}$. The proof is analogous to the proof of Theorem 5.5. Again, the fact that $A_{u}\left(F_{1}, F_{2}\right) \neq 0$ can be deduced from Proposition 6.2.6 in [6] and the results in [8].

Theorem 6.4. Let $F_{i} \in \operatorname{GL}\left(n_{i}, \mathbb{C}\right)$ by such that $\operatorname{Tr}\left(F_{1}^{*} F_{1}\right)=\operatorname{Tr}\left(\left(F_{1}^{*} F_{1}\right)^{-1}\right)=\operatorname{Tr}\left(F_{2}^{*} F_{2}\right)=\operatorname{Tr}\left(\left(F_{2}^{*} F_{2}\right)^{-1}\right)$.

- Denote by $A_{u}\left(F_{1}, F_{2}\right)$ the universal unital $C^{*}$-algebra generated by the coefficients of

$$
X \in M_{n_{2}, n_{1}}(\mathbb{C}) \otimes A_{u}\left(F_{1}, F_{2}\right) \quad \text { with relations } \quad X \quad \text { and }\left(F_{2} \otimes 1\right) \bar{X}\left(F_{1}^{-1} \otimes 1\right) \text { are unitary } .
$$

Then, $A_{u}\left(F_{1}, F_{2}\right) \neq 0$ and there exists a unique pair of commuting universal ergodic coactions of full quantum multiplicity, $\delta_{1}$ of $A_{u}\left(F_{1}\right)$ and $\delta_{2}$ of $A_{u}\left(F_{2}\right)$, such that

$$
\left(\iota \otimes \delta_{1}\right)(X)=X_{12}\left(U_{1}\right)_{13} \quad \text { and } \quad\left(\iota \otimes \delta_{2}\right)(X)=\left(U_{2}\right)_{12} X_{13} .
$$

Here, $U_{i}$ denotes the fundamental corepresentation of $A_{u}\left(F_{i}\right)$.

- $\left(A_{u}\left(F_{1}, F_{2}\right), \delta_{1}, \delta_{2}\right)$ is isomorphic with the $C^{*}$-algebra $B_{u}$ and the coactions thereon given by Proposition 3.13 and the monoidal equivalence $A_{u}\left(F_{1}\right) \underset{\text { mon }}{\sim} A_{u}\left(F_{2}\right)$ of Corollary 6.3.

Remark 6.5. Exactly as in Corollary 5.9, a combination of Theorems 6.2 and 6.4 gives a complete classification of the ergodic coactions of full quantum multiplicity of $A_{u}(F)$ and of the 2-cohomology of the dual of $A_{u}(F)$.

A precise parameterisation of the unitary fiber functors on the quantum groups $A_{u}(F)$ is easy. If $F_{1}, F \in$ $\operatorname{GL}(n, \mathbb{C})$, we write

$$
F_{1} \sim F \quad \text { if and only if there exist unitary } v, w \in \mathrm{U}(n) \text { such that } F_{1}=v F w \text {. }
$$

We study matrices $F \in \operatorname{GL}(n, \mathbb{C})$ satisfying $\operatorname{Tr}\left(F^{*} F\right)=\operatorname{Tr}\left(\left(F^{*} F\right)^{-1}\right)$ up to the equivalence relation $\sim$. It is obvious that for any $\operatorname{such} F$, there exist unique $0<\lambda_{1} \leq \cdots \leq \lambda_{n}$ satisfying $\sum_{i} \lambda_{i}^{2}=\sum_{i} \lambda_{i}^{-2}$ such that $F \sim D\left(\lambda_{1}, \ldots, \lambda_{n}\right)$. Here $D\left(\lambda_{1}, \ldots, \lambda_{n}\right)$ denotes again the diagonal matrix with the $\lambda_{i}$ along the diagonal. 


\section{References}

[1] S. BaAJ \& G. Skandalis, Unitaires multiplicatifs et dualité pour les produits croisés de $\mathrm{C}^{*}$-algèbres. Ann. Scient. Ec. Norm. Sup. 26 (1993), 425-488.

[2] T. BAnica, Théorie des représentations du groupe quantique compact libre $O(n)$. C. R. Acad. Sci. Paris Sér. I Math. 322 (1996), 241-244.

[3] T. Banica, Le groupe quantique compact libre U(n). Commun. Math. Phys. 190 (1997), 143-172.

[4] T. BANICA, Quantum automorphism groups of homogeneous graphs. J. Funct. Anal., to appear.

[5] T. BAnica, Quantum automorphism groups of small metric spaces. Pac. J. Math., to appear.

[6] J. Bichon, Galois extension for a compact quantum group. Preprint.

[7] J. Bichon, The representation category of the quantum group of a non-degenerate bilinear form. Comm. Algebra 31 (2003), 4831-4851.

[8] J. Bichon, Corepresentation theory of universal cosovereign Hopf algebras. Preprint.

[9] J. Bichon, Quantum automorphism groups of finite graphs. Proc. Amer. Math Soc. 131 (2003), $665-673$.

[10] F. BocA, Ergodic actions of compact matrix pseudogroups on $\mathrm{C}^{*}$-algebras. In Recent advances in operator algebras (Orléans, 1992). Astérisque 232 (1995), 93-109.

[11] A. Bruguières, Dualité tannakienne pour les quasi-groupoïdes quantiques. Comm. Algebra 25 (1997), $737-767$.

[12] J.S. Carter, D.E. Flath \& M. Saito, The classical and quantum $6 j$-symbols. Mathematical Notes 43, Princeton University Press, Princeton, NJ, 1995.

[13] S. Doplicher, R. Longo, J.E. Roberts \& L. Zsidó, A remark on quantum group actions and nuclearity. Rev. Math. Phys. 14 (2002), 787-796.

[14] V.G. Drinfel'D, Quantum groups. Proceedings of the International Congress of Mathematicians, AMS, Providence, 1987, pp. 798-820.

[15] P. Etingof \& S. Gelaki, On cotriangular Hopf algebras. Amer. J. Math. 123 (2001), 699-713.

[16] R. Høegh-Krohn, M.B. Landstad \& E. Størmer, Compact ergodic groups of automorphisms. Ann. of Math. (2) 114 (1981), 75-86.

[17] M. Jimbo, A q-difference analogue of $U(\mathfrak{g})$ and the Yang-Baxter equation. Lett. Math. Phys. 10 (1985), 63-69.

[18] M. Landstad, Simplicity of crossed products from ergodic actions of compact matrix pseudogroups. In Recent advances in operator algebras (Orléans, 1992). Astérisque 232 (1995), 111-114.

[19] M. LANDSTAD, Ergodic actions of nonabelian compact groups. In Ideas and methods in mathematical analysis, stochastics, and applications (Oslo, 1988), Cambridge Univ. Press, Cambridge, 1992, pp. $365-388$.

[20] S. MacLane, Categories for the working mathematician. Graduate Texts in Mathematics 5, SpringerVerlag, New York-Berlin, 1971.

[21] M. Rosso, Algèbres enveloppantes quantifiées, groupes quantiques compacts de matrices et calcul différentiel non commutatif. Duke Math. J. 61 (1990), 11-40.

[22] P. Schauenburg, Hopf bi-Galois extensions. Comm. Algebra 24 (1996), 3797-3825. 
[23] R. Tomatsu, Compact quantum ergodic systems. Preprint (2004).

[24] K.-H. Ulbrich, Galois extensions as functors of comodules. Manuscripta Math. 59 (1987), 391-397.

[25] A. Van Daele \& S. Wang, Universal quantum groups. Internat. J. Math. 7 (1996), 255-263.

[26] S. WANG, Ergodic actions of universal quantum groups on operator algebras. Comm. Math. Phys. 203 (1999), 481-498.

[27] A. Wassermann, Ergodic actions of compact groups on operator algebras. I. General theory. Ann. of Math. (2) 130 (1989), 273-319.

[28] A. Wassermann, Ergodic actions of compact groups on operator algebras. II. Classification of full multiplicity ergodic actions. Canad. J. Math. 40 (1988), 1482-1527.

[29] A. Wassermann, Ergodic actions of compact groups on operator algebras. III. Classification for SU(2). Invent. Math. 93 (1988), 309-354.

[30] S.L. Woronowicz, Compact quantum groups. In Symétries quantiques (Les Houches, 1995), NorthHolland, Amsterdam, 1998, pp. 845-884.

[31] S.L. Woronowicz, Twisted SU(2) group. An example of a non-commutative differential calculus. Publ. Res. Inst. Math. Sci. 23 (1987), 117-181.

[32] S.L. Woronowicz, Tannaka-Krein duality for compact matrix pseudogroups. Twisted SU $(N)$ groups. Invent. Math. 93 (1988), 35-76.

[33] S. Yamagami, Fiber functors on Temperley-Lieb categories. Preprint (2004). 